\title{
Intensive use of Lagrangian trajectories to quantify coastal area dispersion
}

\author{
Petton Sébastien ${ }^{1, *}$, Pouvreau Stéphane ${ }^{1}$, Dumas Franck ${ }^{2}$
}

\author{
1 Ifremer, Univ Brest, CNRS, IRD, LEMAR, 11 Presqu'île du Vivier, F-29840, Argenton en Landunvez, \\ France \\ 2 SHOM / STM / REC, 13 Rue de Châtellier CS 92803, 29228, Brest CEDEX 2, France \\ * Corresponding author : Sébastien Petton, email address : sebastien.petton@ifremer.fr
}

\begin{abstract}
:
Coastal waters are subject to great environmental and anthropogenic pressures. The diffusion and the transport of these waters are a key element for environmental, ecological and economic management. There are numerous indicators of hydrological characteristics based on theories of transport time scale. However, these indicators strongly depend on the geographical shape of the studied area and tend to give information after long integration time periods, generally on the order of weeks. Here, to qualify a coastal area's dispersion more precisely, we combined two Lagrangian approaches and estimated a local diffusivity. This paper presents the numerical implementation and the results obtained over a tidally flushed, semi-enclosed water body located at mid-latitude. This new coefficient was estimated using the hydrodynamic model MARS 3D with a barycentric repositioning technique over a tidal period to ensure its reliability. We highlight the existing relationships between local diffusivity and both horizontal and vertical processes. Methodological aspects were analysed based on a reference case (number and distribution of particles, resolution, integration time period). The consistency and sensitivity of the coefficient were studied with different forcing conditions (hydrodynamical and meteorological regimes). In conclusion, our local diffusivity provides a new perspective for understanding the land-sea interface and coastal dispersion and holds potential for future studies of coastal marine ecosystems.
\end{abstract}




\section{Introduction}

Water bodies that are at the land-sea interface (bays, estuaries, lagoons, etc.) are prime sites for (potentially conflicting) human activities (such as transport, aquaculture, fishing and leisure activities) that require conciliatory measures formulated by policy makers. Ocean shores are also of interest to scientists working in various fields (physics, marine chemistry, biogeochemistry, ecology, etc.). For instance, larval transport, dispersal and population connectivity of marine species (mainly fishes and invertebrates) in coastal areas are topics of growing interest for marine ecology because they constitute key processes for population dynamics, gene flow, the spread of invasive species, marine ecological restoration, the establishment of marine protected areas (MPAs) and other related topics (e.g. Pineda et al. 2007).

Coastal areas are, on the one hand, more or less connected to the open ocean and thus renewed by oceanic waters, and, on the other hand, they collect watershed waters of very different and distinctive characteristics (in terms of salinity, solid particle load, concentration of various chemical substances such as nutrients, heavy metals, micro-plastics, etc.), which also constitute a source of water renewal. At least superficially, coastal waters can be qualified as buffer or mixing areas.

Going beyond this simplified picture requires a more detailed description of the way oceanic and continental waters mix and how the spread of this water mass is driven by various hydrodynamic processes and their interactions within basins of relatively complex geometries and bathymetries. These interactions occur over a wide range of spatial and temporal scales, even at high frequency (from hours to months). They appear too complex for certain applied purposes and have raised the question of using deterministic geographical indicators for a long time.

When the Water Framework Directive was implemented in the early 2000s, it was required to split water masses into "homogeneous" coastal areas whose monitoring would be affordable. Lazure et al. (2008) provided a guide to determine homogeneous units based on tidal residual current intensity and the Simpson and Hunter (1974) criteria.

Long before this period, as early as the 1970s (Bolin and Rodhe 1973; Zimmerman 1976), the scientific literature contains many studies that focused on providing typologies for water bodies. Over the years, the methods have benefited from the improvement of observation facilities (LaCasce and Ohlmann 2003; Brown et al. 2009; Rypina et al. 2016) and modelling capacities, with ever-increasing computing power (Sawford 2001; Haza et al. 2008). These improvements now allow for an extensive numerical approach that was not possible some years ago, particularly Lagrangian approaches that can track millions of numerical particles (van Sebille et al. 2018; Jo et al. 2019).

Most of these approaches tightly link spatial and temporal scales more or less implicitly (Monsen et al. 2002; Delhez et al. 2014; Cucco and Umgiesser 2015; Viero and Defina 2016). The time scale is often provided in the framework of the Constituent-oriented Age and Residence time Theory (CART, www.climate.be/cart), being called residence time (Delhez et al. 2004), exposure time (Delhez 2006) or water age (Deleersnijder et al. 2001). These indicators rely either on a particular spatial scale (a bay, an estuary, a grid) at which particular processes will renew the water mass (through open - ocean - connected boundaries or through runoff inputs) or transport water mass across the considered spatial

entity; or either on well-identified source of a given water mass whether it is coming across a well-identified boundary, 
a volume or a singular point (tip end of an estuary). They could possibly be used to figure out the transport in a specific direction, such as the vertical one (Meier 2005; White and Deleersnijder 2007; Bendtsen et al. 2009).

This kind of indicator - even called "local" - fundamentally relies on the definition of a more global feature that controls water volume (flushing time or $e$-flushing time as used by Plus et al. (2009)) or the point at which the renewed water mass enters the considered body. Numerical methods have also been developed to decompose the studied area into several subdomains (de Brauwere et al. 2011). In this way, Mouchet et al. (2016) proposed the notion of partial ages to identify the fate of some water bodies, for instance the transport routes from the ocean's surface to deeper layers. Another kind of method to split the domain under study consists in using the hydrodynamic connectivity in a biological context (Kool et al. 2013). It can be combined with machine learning techniques to build a theoretical network for marine conservation or restoration (Kininmonth et al. 2010; Rossi et al. 2014; Thomas et al. 2014).

Recently, Fiandrino et al. (2017) presented temporal indicators in another way, using the tight relationships between the temporal and spatial scales of renewal to provide a mixing volume with respect to a specific, given time scale relevant to the specific question at hand. In fact, their indicator is well suited to low-energy environments where seawater renewal is considered as a good proxy for the environmental gradient.

All of these approaches generally focus on a pre-defined targeted area, usually semi-enclosed even if they are formally devised to deal with any ocean body configuration. The fact that they are built around a peculiar control domain unescapably selects some spatial and temporal scale. Besides, they could not provide with an intensive property of the medium which is one of the intended goals hereafter.

In order to obtain a complementary and more local view that relies on the capability of the water masses to mix or disperse, Capet et al. (2008) sought to quantify enhanced diffusivity due to sub-mesoscale turbulence at a regional scale (i.e. the continental shelf) using an Eulerian approach. Under certain fundamental assumptions (isotropy, stationarity, existence of a Reynolds decomposition) that must be met to remove the skewed fluxes, they computed a diffusivity as follows:

$$
k=-\frac{\langle\widetilde{\boldsymbol{u}} \tilde{T}\rangle \cdot \nabla_{\mathrm{h}}\langle T\rangle}{\left|\nabla_{h}\langle T\rangle\right|^{2}}
$$

It is based on the field tracer dynamics of temperature (here denoted T) and a Reynolds decomposition (<.>, ) of all the fields. These assumptions may be met at the scale they worked at, but are harder to meet near the coast where there are high levels of anisotropy and non-stationarity due to complex basin geometry and topography along with, strong tidal forcings.

Developed in the communities that analyse drifter data (LaCasce 2008) and oceanic models (van Sebille et al. 2018), other methods rely on the fate of an ensemble of particles to quantify mean diffusivity and extra Lagrangian diagnostics. Some recent studies (Rypina et al. 2016; Suara et al. 2017) provide approaches and results on which our study is based. Here, we used this framework to assess local 2D relative dispersion (i.e. mean over the vertical) in coastal areas at small spatial and short temporal scales. It is hard within such a realistic framework to separate and quantify the various contributions to the holistic dispersion in fine experienced by any dissolved materials. Ridderinkhof and Zimmerman (1992) pointed out at least three of them: (a) The vertical shear dispersion (interplay between vertical turbulence and 
vertical velocity shear) which horizontal equivalent can be formulated as $K_{H}=\alpha \frac{H^{4} M^{2}}{K_{Z}}$ where $H, M, K_{Z}$ stand, respectively, for the water column depth, the (vertical) shear frequency and the vertical diffusivity. $\alpha$ is a nondimensional parameter which might be of $O(1)$. This gives at least an order of magnitude of this contribution; (b) The horizontal shear dispersion is due to the interplay between the horizontal turbulence and the horizontal velocity shear. This contribution is a priori thought to be weak with respect to the two others as long as the depths increase. But, in the far end of the sheltered coves, where the depths dramatically decrease, it may become the dominant component; (c) The dispersion resulting from the combination of the residual field and the instantaneous velocity that fuelled it. This part may overwhelm the previous contributions as illustrated by Ridderinkhof and Zimmerman (1992). This contribution is implicitly captured as long as the approach accounts for the full span of time and spatial scales (from subtidal to supertidal scales).

The proposed method to cope with some of the various contributions afore mentioned uses a Lagrangian approach whose fundamentals are presented in $\$ 2.1$. It is implemented over a typical and relatively representative semi-enclosed area described in $\$ 2.2$ using a numerical model whose setup is given in $\$ 2.3$ according to our experimental plan (\$2.4). The results given in $\$ 3.1$ show the links between this dispersion and some practical characteristics such as tidal excursion (§3.2). The robustness of the dispersion capabilities is assessed with respect to temporal and spatial scales ( $\$ 3.3$ and §3.4). Some insights into the response of the indicator are assessed with respect to hydrodynamical regimes and meteorological forcings ( $\$ 3.5$ and $\$ 3.6)$.

\section{Materials \& Methods}

The method used to assess a local diffusivity lies on particle tracking and common statistical concepts that are detailed below. We focused on a particular macrotidal environment, and explored how to filter out the tidal signal so as to provide a coherent picture of the dispersion fields. To do so, we used a barycentric repositioning method that gives tidal residual current fields (see $\$ 2.1$ below). This approach can nevertheless be generalised as long as this particular step is ignored of fitted to non-tidal environments. In such environment one has to adapt the repositioning procedure to account for the low-frequency displacements that may induce net transport. It nonetheless requires additional care because physical indicators - which may be used straightforwardly in non-tidal environment - are filtered out.

The Lagrangian approach had rather been used for the purpose of this study mostly because of its agility with respect to the nature of its data; further, as long as we restricted our study to a short time range (from one to few tidal cycles) we are unlikely to face the problem of statistical meaninglessness due to large scattering of the initial particle clouds. The unquestionable benefit of the lagrangian approach lies in the fact that from an initial homogeneous sampling we can $a$ posteriori develop many sampling strategies (see hereafter for example the scale of the initial cloud considered (noted DES), the sampling step for aggregation (SSF), etc...). It would even have additional benefits in the case of strong interaction of the particles with their environment as for an individual based model approach which is clearly not the case here for purely passive particles that mimic water parcels. Last, the main point is that we lack an objective method to properly choose the appropriate and optimal number of particles we should use not to miss any crucial structure and catch all the core variance. Nevertheless, we adopted an empirical approach and performed partial convergence tests that are explained in further detail (see $\$ 2.1$ ). 
This local approach gives a mean of the vertical dimension of dispersion, thus being a measure of the dominant combination of horizontal advection and vertical mixing. This method is applied using a high-resolution numerical model set up over a realistic area to mimic coastal flows (including the level of mixing) with a high level of realism. The bay under study (see \$2.2) shows a wide range of variability due to strong tides and complex bay geometry. The numerical model used is a classical one (primitive equation discretised with finite differences and a structured grid in a vertical sigma coordinate framework) and includes an online particle track computation capability allowing almost perfect sampling $(\S 2.3)$.

\section{Estimation of the local diffusivity}

The local diffusivity is estimated by tracking Lagrangian trajectories. We estimated the relative diffusivity for each group of N particles based on the single-particle dispersion tensor (LaCasce 2008; Kamenkovich et al. 2009; Döös et al. 2011; Rypina et al. 2016), which represents the spreading of the particle's cloud between its initial and final position relative to its centre of mass:

$$
\begin{gathered}
D_{x x}(t ; y)=\frac{1}{N} \sum_{n=1}^{N}\left[\Delta x_{n}(t)-\Delta X(t)\right]^{2} \\
D_{x y}(t ; y)=\frac{1}{N} \sum_{n=1}^{N}\left[\Delta x_{n}(t)-\Delta X(t)\right]\left[\Delta y_{n}(t)-\Delta Y(t)\right] \\
D_{y y}(t ; y)=\frac{1}{N} \sum_{n=1}^{N}\left[\Delta y_{n}(t)-\Delta Y(t)\right]^{2}
\end{gathered}
$$

where $\Delta x_{n}$ and $\Delta y_{n}$ are respectively the zonal and meridional displacement of the $\mathrm{n}^{\text {th }}$ particle from its initial position, and $\Delta X(t)=(1 / N) \sum_{n=1}^{N} \Delta x_{n}(t)$ and $\Delta Y(t)=(1 / N) \sum_{n=1}^{N} \Delta y_{n}(t)$ are the ensemble-mean displacements. Because real flows often exhibit anisotropic transport properties, particles are spread along a preferential direction, forming an approximate ellipse. To account for this likely anisotropy, the system is diagonalised with a rotation of an angle $\theta$ between the zonal direction and the direction of the fastest spread (O'Dwyer et al. 2000; Rypina et al. 2012). The dispersion in the major direction (denoted by $\xi$ ) and its perpendicular (denoted by $\eta$ ) are expressed as:

$$
\begin{gathered}
D_{\xi}=D_{x x} \cos ^{2}(\theta)+D_{y y} \sin ^{2}(\theta)+D_{x y} \sin (2 \theta) \\
D_{\eta}=D_{x x} \sin ^{2}(\theta)+D_{y y} \cos ^{2}(\theta)-D_{x y} \sin (2 \theta)
\end{gathered}
$$

$\theta$ is estimated by maximising equation (4), i.e. by choosing $D_{\xi}$ to be the largest value (Rypina et al. 2012), which leads to the following equation:

$$
\tan (\theta)=\frac{D_{y y}-D_{x x}+\sqrt{\left(D_{y y}-D_{x x}\right)^{2}+4 D_{x y}}}{2 D_{x y}}
$$

Thus, the diffusivities in the major direction and its perpendicular are expressed as $K_{\xi}(t)=\frac{1}{2} \frac{\partial D_{\xi}}{\partial t}(7)$ and $K_{\eta}(t)=\frac{1}{2} \frac{\partial D_{\eta}}{\partial t}$ (8) and are computed over an integration period of one tidal cycle. The ratio $R_{D}=D_{\xi} / D_{\eta}(9)$ is referred to as the anisotropy coefficient. 
This assessment of diffusivities relies on an assumption of stationarity of the temporal derivative of the ellipse size with respect to time, which is supposed to be reached after a ballistic regime phase (Rypina et al. 2016). However, it is unlikely to be reached over a long time period because of the large tidal residual current (see Fig. 3) and strong spatial heterogeneity of the instantaneous tidal currents. We will restrict the approach to short time scales (i.e. a few tidal cycles) to remain under reasonable stationarity assumption.

We homogeneously sowed the domain with particles that are evenly distributed on the horizontal and vertical directions at the initial time. The spatial discretisation was linked to the size of the model grid, which in a sense is the typical size of the cut-off spatial scale. We chose to put particles within each mesh grid according to the following distribution: each $\Delta x / 5$ in the horizontal direction and on four vertical levels (Fig. 1b). This is what we will call the sampling scale of the flow (SSF). The second scale we introduced is the dispersion estimation scale (DES): this is the size of the particle clouds we chose a posteriori to estimate the diffusivity (blue circles in Fig. 1b). The Lagrangian approach makes it possible to make independent choices for these two scales (SSF and DES) possible: the only constraint being to obtain a sample large enough for the latter scale (DES) to get a robust estimation of the variances given from equation 1 to 3 . 100 particles per grid cell (ppgc) were finally used providing about 5000 pairs for the sums of the equations 1 to 3 . Besides, the relevancy of that amount of ppgc was empirically strengthened with a convergence test (not shown): it has been performed over a subdomain (to reach very high particle density that would be unreachable considering the whole domain) with increasing sets of particles (from 5 up to 1600 ppgc). The highest manageable ppgc number for the whole domain (with respect to CPU cost) above which the results vary only a few percent was retained. The convergence curves show that with $100 \mathrm{ppgc}$, we capture more than $95 \%$ of the variance obtained with 1600 ppgc.

To get rid of the initial instant of particle release with respect to tidal phase whose impact can be very large (see Fig. 2 in Bailly du Bois and Dumas 2005), we used a barycentric repositioning method to provide a more coherent picture of the diffusivity field. It has been tested and described by Salomon and Breton (1991) and Salomon et al. (1996) to give tidal residual current fields whose computation is based on the same particle-tracking approach. The main point (Fig. 1a) is to retain the intrinsic information from each particle track that is not only tightly linked to its initial position and phase release, but also more generally based on the shape of the track and its centre of mass. The impact on the computation of the tidal residual current is clearly illustrated on Fig. 3 which shows the way we recovered coherency over fields (compare Fig. 3a, without Lagrangian repositioning -- each residual current kept positioned at its initial position whatever its release instant -- with Fig. 3b, with Lagrangian repositioning done for each particle).

After the repositioning step for all the particles, we obtain a spatial distribution of the barycentre (one per particle track) which is not fully controlled and may have some gaps. To fill in the domain as much as possible and obtain the best final resolution for the diffusivity grid estimation, we released the same particle fields every hour for an even coverage of the tidal cycle and multiplied the possible trajectories, i.e. 13 releases during the entire lunar $\mathrm{M}_{2}$ tidal wave cycle. We spread the statistical support for the estimation of dispersion (which are circles of size DES) over every particle centre relative to SSF (Fig. 1b). The overlapping distance between two consecutive circles of size DES is denoted hereafter as OD.

Fig. 1 
The last step of the diffusivity field reconstruction was the estimation of the diffusivity at the nodes of a regular output grid whose cell size is that of the hydrodynamic model. The estimation of the dispersion coefficient is just an evenly weighted mean over the whole set of final centroids located in each grid cell. We ensured that all the cells of the final grid were filled properly to have enough samples to compute a mean (roughly more than 10 samples within each grid cell).

\section{Study site}

To test the concept, we implemented it over a real geographic site that undergoes strong tidal currents, has a very complex geometry and topography, a high level of spatial heterogeneity, and is otherwise relatively well known, being the subject of a large amount of oceanographic studies.

This reference case is the Bay of Brest, located at the western end of Brittany (France). It is a semi-enclosed marine ecosystem, spanning over $180 \mathrm{~km}^{2}$. It is connected to the Iroise Sea (Atlantic Ocean) through a $1.8 \mathrm{~km}$ wide by $6 \mathrm{~km}$ long and roughly $50 \mathrm{~m}$ deep inlet (called the Goulet de Brest, see Fig. 2). With less than 15\% of its total surface below $20 \mathrm{~m}$ and $50 \%$ above $5 \mathrm{~m}$, the Bay of Brest has an average depth of $8 \mathrm{~m}$. It is a shallow bay with intertidal flats and several sheltered coves (e.g. Auberlac'h, Roscanvel, Fret, Daoulas, etc.) as a result of its relatively fractal coastline. This macrotidal coastal area is characterised by a semi-diurnal dominated tide with a tidal range that goes from 1.2 to $7.3 \mathrm{~m}$. The tidal currents can reach up to $3 \mathrm{~m} \cdot \mathrm{s}^{-1}$ in the Goulet and are in quadrature-phase relative to the surface elevation. The mean volume at mid-tide behind the Goulet de Brest is roughly 2 billion $\mathrm{m}^{3}$. The back-and-forth flow at each tide prevents any stratification (Le Pape and Menesguen 1997), except deep in the estuary mouths. There are four main areas: the centre of the bay adjacent to the Goulet, the south-eastern part of the bay and the two estuaries of the Aulne River and the Elorn River. The hydrology is dominated by freshwater runoffs, with 63\% coming from the Aulne River (1875 km² watershed), 15\% from the Elorn River $\left(385 \mathrm{~km}^{2}\right), 8 \%$ from the Douffine River $\left(139 \mathrm{~km}^{2}\right)$ and $5 \%$ from the Mignonne River $\left(110 \mathrm{~km}^{2}\right.$ ) (Auffret 1983). The enclosed part of the bay is not affected by oceanic swells. However, strong south-westerly winds (over $15 \mathrm{~m} \cdot \mathrm{s}^{-1}$ ) may generate wind waves with significant heights of more than $80 \mathrm{~cm}$ in the northern part of the bay and in the Daoulas Cove (Guillou 2007; Petton 2010). Nevertheless, any mixing generated by waves due to topographic breaking was not accounted for here. Due to its complex geography, the Bay of Brest's current patterns show strong spatial heterogeneity with a non-stationary phase thereby meeting the previously mentioned prerequisites. It is a very energetic coastal area characterised by complex mixing mainly attributed to one process (the tide), which is thus reproducible at every tidal cycle and all year long.

\section{Fig. 2}

The investigation of such realistic flows and especially the way to determine the various processes at play in mixing is only possible using a numerical model whose characteristics - although relatively common in the literature - are exposed hereafter.

\section{Hydrodynamic model}

In this study, we set up the hydrodynamic "Model for Application at Regional Scale" (MARS 3D) (Lazure and Dumas 2008) over the whole Bay of Brest (geographic limits: $48.20^{\circ} \mathrm{N}-48.44^{\circ} \mathrm{N}$ and $4.09^{\circ} \mathrm{W}-4.72^{\circ} \mathrm{W}$ ). It solves the NavierStokes equations under the hydrostatic and Boussinesq assumptions (Blumberg and Mellor 1987) over a curvilinear grid 
with a horizontal resolution of $50 \mathrm{~m}$ and $20 \sigma$-layers over the vertical. Bathymetry is interpolated from a combination of different digital terrain models (SHOM, Ifremer, IGN) including the latest LIDAR acquired data to represent the intertidal slope for wetting-drying simulations. The model is forced by the sea-surface elevation along the open boundaries obtained from a wider 2D model with a $250 \mathrm{~m}$ resolution over the whole Iroise Sea that encompasses the targeted area and forced by harmonic components from the SHOM CST-France model (Le Roy and Simon 2003). Freshwater inputs for the four main rivers were taken from the HYDRO database (http://www.hydro.eaufrance.fr/) and corrected with corresponding watershed rates.

The surface drag coefficient was set uniformly to obtain the optimum validation of the currents in the Bay of Brest. However, the bottom drag coefficient relied on the Prandtl theory of the bottom boundary layer and its logarithmical shape. It includes in its formulation a classical roughness scale, which was spatialised according to a recent sedimentological map (Gregoire et al. 2016).

The horizontal turbulent closure consists of a Laplacian operator with a constant turbulent viscosity coefficient. This coefficient was set to $0.5 \mathrm{~m}^{2} . \mathrm{s}^{-1}$. Knowing that a large amount of the effective viscosity lies within the numerical schemes, this value was empirically chosen; it is the lowest that properly dissipates the small scales due the diffusivity of the numerical advection schemes. The vertical turbulent closure is performed using the Generalised Length Scale parametrisation with a two-equation k- $\epsilon$ model (Umlauf and Burchard 2003; Warner et al. 2005). It is assumed that density effects on circulation and turbulence are weak. This assumption holds relatively well except inside the estuary or in their mouths where some haline stratification may occur. However, we still accounted for the water mass inputs from the rivers.

The model has demonstrated its ability to reproduce the main characteristics of oceanic flows (tidal amplitude and phase, 3D currents) with a high level of realism. It was for this reason that this model was compared with various datasets (tidal gauge, ADCP current, temperature and salinity from buoys) from many studies in the area (Petton et al. 2016, 2018). This high degree of realism is due to the fact that the flows are driven by the tide whose large-scale features are easily captured.

For the Lagrangian approach, we used a trajectory computation capability fully coupled to the hydrodynamic model. Thus, the Lagrangian trajectories were simulated online simultaneously with the computation of the dynamical fields (velocity, sea-surface elevation, etc.). A random walk function was implemented within the particle-tracking model to simulate subgrid-scale vertical diffusion based on an Euler scheme as described by Visser (1997) and modified by North et al. (2006). Despite more accurate numerical schemes have been developed (Gräwe et al. 2012; Shah et al. 2013; van Sebille et al. 2018), especially to overstep sharp pycnoclines, it was better to use a less precise but low cost calculation approach to deal with a large number of particles. This simplification is rather acceptable as long as the area does not exhibit a complex structure of the vertical mixing coefficient. No horizontal diffusion was introduced in the initial reference experience. Particles were allowed to ground on the shoreline because the MARS 3D model manages the wetting-drying of intertidal areas. The duration of the tracking simulation was set to exactly one tidal cycle to properly filter out the tidal signal.

With 100 passive particles per mesh cell inside the bay, this represents more than 7 million trajectories to compute at each time step. We dealt with this large number of particles using a massive parallelisation approach based on MPI: 
using $336 \mathrm{CPU}$ cores, we simulated a tidal cycle within 10 hours. The numerical estimation of the coefficient and the plotting of the figures were performed using Python 3.6 software.

\section{Case scenario}

We set out to test our method and attempt to explain the way different processes act on dispersion. We therefore performed many numerical experiments. Each simulation was performed according to the following protocol: a hydrodynamic spin-up run was performed for 5 days (10 times longer than the period required for the tidal wave to propagate from the entrance of the bay to its far end) before the release of the particles.

To obtain various tidal regimes (especially tidal excursion interaction with respect to bathymetric or coastline features scales), we performed three categories of simulation with respect to the tidal range. Our reference simulation was the mean tidal range ( $4.3 \mathrm{~m}$ tidal range - coefficient 70 on a scale varying from 20 to 120). We also investigated mean spring tides (5.9 $\mathrm{m}$ tidal range - coefficient 95 on the same tidal scale) and mean neap tides ( $2.7 \mathrm{~m}$ tidal range coefficient 45). The tide was set as a perpetual cycle. All of these simulations were performed without any atmospheric forcings.

The Bay of Brest is located at mid-latitude and thus is subject to highly variable and sometimes intense meteorological forcings. We also investigated the impact of wind. For this reason, we restricted the simulation to mean tide conditions and set up different typical constant winds of 5 and $10 \mathrm{~m} . \mathrm{s}^{-1}$ over a tidal cycle for the two major directions of seasonal winds, i.e., south-westerly and north-easterly winds. These conditions were chosen based on the average wind from AROME analysis data from 2008 to 2017 (Météo-France $2.5 \mathrm{~km}$ ). For simplification, the wind was set as stationary and homogeneous over the whole model domain.

Last, but not least, in the reference case (mean tide and no atmospheric forcings), we investigated some methodological aspects of our approach by modifying the various scales involved (numerical grid model, SSF, DES, etc.).

\section{Results}

Several numerical studies focused on modelling transport in the presence of strong tidal flow. Solutions exist to obtain a tidally average solution for long-term computations in coastal waters (Nihoul and Ronday 1975; De Kok 1994). The Lagrangian current framework has been successfully used to determine the residual current (Ridderinkhof and Zimmerman 1992; Ridderinkhof and Loder 1994; Delhez 1996; Wei et al. 2004; Liu et al. 2012; Charria et al. 2013). In that spirit, the importance of the barycentre repositioning technique has been well described in Salomon et al. (1996) and used in Muller et al. (2009). A comparison of a given field plotted with and without the final repositioning is given in Fig. 3 with the estimation of tidal residual currents for mean tide conditions. When the tidal residual currents are located at their initial position, the main flow is oriented towards the ocean but does not necessarily flow along the coastline. Residual currents can come directly from the shore, i.e. they do not have any realistic meaning. On the other hand, the barycentre repositioning technique revealed logical eddy structures, especially with the anticyclonic eddy situated at the entrance of the bay. Moreover, the standard deviation of the mean over the 13 time-release runs decreased significantly (not shown here). This strengthened the physical value of the field. Nevertheless, there are different 
locations where the eddies hit the coast. These occurrences can be explained by geographic elements near points or dikes.

\section{Fig. 3 a, b}

\section{Reference case results}

The reference case (mean tide, no atmospheric forcings) is shown in Fig. 4. It shows the numerical estimation of the local diffusivity (in $\mathrm{m}^{2} . \mathrm{s}^{-1}$ ) in the fastest spreading direction $K_{\xi}$ (as defined from $D_{\xi}$ in eq. 7) and the anisotropic ratio $R_{D}$ (see eq. 9). It shows only part of the whole model grid (see Fig. 2). It was assessed with an SSF of $10 \mathrm{~m}$ and $50 \mathrm{~m}$ and an OD of $10 \mathrm{~m}$. The diffusivity was estimated over the native hydrodynamical grid whose resolution is $50 \mathrm{~m}$.

\section{Fig. 4 a, b}

The diffusivity ranged from $10^{-2} \mathrm{~m}^{2} \cdot \mathrm{s}^{-1}$ to $10^{2} \mathrm{~m}^{2} \cdot \mathrm{s}^{-1}$ spanning over four orders of magnitude. The highest values $\left(250 \mathrm{~m}^{2} . \mathrm{s}^{-1}\right)$ were reached in the central area of the bay, the most exposed to strong tidal currents and the deepest area. It significantly decreased towards the areas (southeast and northeast ends) less connected to this central area and even one order of magnitude beyond in the sheltered coves such as the Fret, Daoulas and Roscanvel coves, where it dropped down to $10^{-2} \mathrm{~m}^{2} . \mathrm{s}^{-1}$ locally. Furthermore, these minima were reached over rather shallow areas. Such low levels are very likely due to the fact that we omitted the horizontal shear dispersion which may be the main driving process when the water column height faint.

Interpreting these results in terms of mean displacement (as seen by a random walk in two dimensions) gave displacements that ranged between $40 \mathrm{~m}$ and $4 \mathrm{~km}$ over a tidal cycle. The upper limit was observed in a significant part of the bay (roughly 12.5\%) and was even commensurate with tidal extension (see Fig. 6a).

This reference diffusivity estimation given in Fig. 4a shows clear and sharp structures that are well delimited, giving a picture of very contrasted areas over very short spatial scales. The diffusivity can increase by a factor 100 over spatial scales shorter than $1 \mathrm{~km}$. This suggests that there are some confined or at least rather sheltered areas in close proximity to highly dispersive areas. This proximity raises the question of how and how much they are connected at time scales longer than the tidal cycle and how robust these structures are with respect not only to the hydrodynamic regime (tidal amplitude, meteorological forcing), but also with respect to time. These are addressed below.

In addition, the magnitude of the anisotropic coefficient (Fig. 4b) was greater than 2 in more than $98 \%$ of the study domain. The ratio reached more than 10 in many places where the flow is highly constrained in a specific direction by the shape of the basin; e.g. in the Goulet and more generally along the coasts. Given that the dispersion ellipses (Fig. 1a) are rather flattened everywhere, we focused on the maximum coefficient $K_{\xi}$.

To try to determine the way these diffusivity values may be related to physical processes, we carried out the same experiment, but used the barotropic component of the current to transport the particles; this component is simultaneously available in the numerical model (i.e. because based on a mode splitting method). This approach involved switching off the vertical diffusivity because we were no longer within the scope of the experiment for the vertical processes. In these conditions, the diffusivities obtained (not shown) were from 1 to 100 times weaker than the one shown in Fig. 4a. They look similar in deep and highly energetic areas where they were greater than $10 \mathrm{~m}^{2} . \mathrm{s}^{-1}$ in the reference simulation. They were much weaker in the sheltered areas exposed to weak tidal currents, where the shear due to the bottom 
boundary layer may cover a significant part of the whole water column. This situation highlights the fact that the dispersion mechanisms are generally 3D, strongly coupling differential (over the vertical) advection and intense vertical mixing.

This intense vertical mixing makes the numerical experiment virtually insensitive to the initial vertical distribution of the particles within the cell. We performed various trials, between fully random and fully evenly distributed over the vertical, none of which had any significant impact on the final estimated field. The median (over the tidal cycle and the vertical) vertical turbulent diffusion coefficient in Fig. $6 \mathrm{~b}$ ranged between $10^{-4} \mathrm{~m}^{2} \cdot \mathrm{s}^{-1}$ and $1 \mathrm{~m}^{2} . \mathrm{s}^{-1}$. These values are high enough to fully mix the deepest water column within 2 to 3 hours. This is in line with the fact that the initial vertical distribution has no impact on the estimated diffusivity.

Previous works demonstrated that the tidal-phase release time of particles could have a significative impact for quantification of transport time scale (de Brye et al. 2012; Andutta et al. 2016), especially for tidal shallow estuary forced by river discharge conditions. For the bay of Brest, some dissimilarities exist between different time-releases but the main structures remain unchanged showing slight modifications far below an order of magnitude. This is one of the benefits of the barycentric repositioning in a macro-tidal environment with weak river flows. For each of the 13 releasestime, the number of estimations per mesh decreases and a specific area started to suffer from a lack of data as we do not completely control the barycentre of each patch. So, the tidal averaging solution allows to fill the map with as many estimations as possible and to slightly smooth the signal.

\section{Effect of shear diffusivity}

The lowest diffusivity values (below $10^{-1} \mathrm{~m}^{2} . \mathrm{s}^{-1}$ ) found for the reference case are clearly unrealistic. Our experiment, is only addressing part of the dispersion. It does not account for any explicit horizontal dispersion. Processes that result from the subgrid scale of the model are generally simulated with explicit diffusivity.

To evaluate the impact of the horizontal shear dispersion, we made an experiment by adding a horizontal random walk scheme with a diffusivity coefficient of $K_{H}=1 \mathrm{~m}^{2} \mathrm{~s}^{-1}$. This value is based on an estimation according to Okubo's parametrisation scheme (1971): $K_{H}=\alpha \Delta^{1.15} \mathrm{~m}^{2} \mathrm{~s}^{-1}$ where $\Delta$ stands for the space scale considered (here the model grid cell size) and $\alpha$ a coefficient fixed with a validation process based on in-situ data ( $\alpha=0.011$ in our case). In order to compare the final diffusivities with the reference case shown in Fig. 4a, we plotted in Fig. 5. the local diffusivity minus the explicit horizontal diffusivity value imposed. The explicit value thus added appears to have an effect of floor value as long as the structure of this new dispersion field is fully superimposable to the original one. The diffusivity coefficient computed with this additional horizontal explicit dispersion is hardly larger everywhere than the reference value except in the far end of the sheltered coves, where undoubtedly the dispersion is underestimated in the original approach. It may allow us to think that the horizontal shear (or explicit) dispersion is dominant in these shallow water areas and should not be neglected. Last, the horizontal explicit dispersion has a blurring effect of the whole field noticeable by comparing Fig. 4a and 5. However, there is no impact on the standard deviation estimated over the 13 release times whose values remain in the same order of magnitude than the reference case.

Fig 5 
In this way, our approach does not account for all the components of the dispersion but catches the major contributions almost everywhere through the domain. Without this explicit horizontal diffusivity, the method is efficiently selective to highlight weak and strong dispersion areas and gives a relevant big picture of the area.

\section{Link with tidal movements and vertical mixing}

As underlined above, the way the diffusivity relies on some characteristics of flow is not straightforward. We hereafter attempt to relate it to the tidal excursion and the vertical diffusion coefficient, which are two main characteristics of vertical mixing and the spatial length over which vertical displacements may occur.

The local diffusivity is compared with the tidal excursion length plotted on Fig. 6a for the reference case (i.e. mean tide and no meteorological forcing). The tidal excursion length was estimated straightforwardly from the same numerical simulations for each particle, smoothed with the barycentre repositioning technique and averaged over the 13 timerelease simulations.

The tidal excursions extended from less than $1 \mathrm{~km}$ in the far end of the coves to up to $20 \mathrm{~km}$ in the straits. The tidal excursion was larger where the flow is driven by the coastline (within estuaries). There is some degree of dissymmetry between the northern and the southern part of the bay: the excursions were significantly smaller in the northern part, apparently delimited by a very sharp transition. In the southern part of the bay, the tidal excursions were larger and went deeper. The difference is attributable to the fact that the tidal prism in this southern branch was significantly longer than the one in the north due to bathymetric shapes.

There are obvious similarities between both maps (Fig. 4a and 6a) and the probability density function summarises this similarity in Fig. 5c, highlighting the relationships between the tidal excursion and diffusivity. First, there is no clear univocal link between these two quantities: unsurprisingly, there is a more or less linear relationship, where tidal excursion increases with the diffusivity. Aside from this linear relationship, two additional kernels indicate a different link between diffusivity and tidal excursion. The spatial distribution of the points within each kernel does not show any clear evidence of tracks with a particular process or relationship. This distribution highlights the high level of nonlinearity and the scales of heterogeneity present within the Bay of Brest. Additional simulations of various tidal regimes (mean neap and spring tides) were carried out; the correlation approaches near-linear behaviour by aggregating all the kernels of the probability density function along the linear relationships (not shown) while the tidal regimes get weaker. The scaling equation can then be written as $K=l . u\left(\mathrm{~m}^{2} . \mathrm{s}^{-1}\right)$, where $l$ is the tidal excursion and $u$ is a scale for the horizontal velocity (chosen as the mean current along the tidal excursion); it is all the more relevant as for the mean spring tide conditions.

Given that we observed extra kernels in the probability density function, the diffusivity $K$ cannot be fully explained by a single quantity, but by a set of quantities. We pointed out above the link with the vertical turbulent mixing coefficient $k_{z}$. The vertical turbulent mixing coefficient (median over the vertical axis and the tidal cycle) is shown in Fig. 5b. This mean coefficient ranges from $10^{-3} \mathrm{~m}^{2} \cdot \mathrm{s}^{-1}$ in the sheltered coves to almost $1 \mathrm{~m}^{2} . \mathrm{s}^{-1}$ in the central bay swept by the tidal prisms at each ebb and flood cycle. The mean coefficient clearly exhibits patterns similar to the diffusivity fields, e.g. the enhanced central part of the bay, the north/south asymmetry, the weak far end of the bays, etc. It is likely to explain a significant part of the horizontal dispersion variance, but no clear explanation can be found in the probability 
density function between them in Fig. 6d. Lastly, the diffusivity is also strongly correlated with the instantaneous tidal currents. We observed large values near the strait and a good correlation with the local current intensity.

Fig. 6 a, b, c, d

\section{Evolution over time integration}

We previously raised two questions on the robustness of the dispersive structure and the level of dispersion with respect to time. Up to now, they were both estimated over a single tidal cycle to filter out the effect of tide exactly and to perform a proper barycentric repositioning. To investigate these two points, simulations were performed over two, three and four tidal cycles of the reference tidal situation (i.e. a perpetually repeated mean tidal cycle). The results are given in Fig. 7.

Fig. 7 a, b, c

First, the main structures of the local diffusivity persisted everywhere: places with rather weak dispersive capability continued to be so after two days (four tidal cycles). Places with high dispersive capability tended to spread and the overall level of dispersion tended to increase slightly over the entire bay. The main deformation occurs in the Daoulas Cove with an amplification of magnitude 10 compared with the reference case. This means that after a single tidal cycle, a stability plateau was not reached and the dispersive coefficient fields kept growing. This result highlights the difficulties met here due to the strong spatial heterogeneity and the fact that residual transport (linked to the tidal residual) is hardly separable from pure dispersion. Another explanation involves the repositioning technique, which encompasses the residual circulation after each tide cycle. There is a lack of data in some places near the coastline, which is due to the flushing of the particles outside the bay. The constant renewal leads to a rapid decrease in the number of cloud estimations used for every final mesh cell.

\section{Methodological aspects}

The number of passive particles needed to capture the behaviour of a water body can become critical in dispersive areas. From a methodological point of view, because the local diffusivities can be estimated offline after the run, the number of particles does not impose a given number of clouds. Therefore, a particle can be used several times in different clouds that overlap. The clouds' initial centres are located on a sub-grid compared with the hydrodynamic grid and their DES radii are equal to the horizontal resolution of the model $d x$. The sub-grid has a horizontal resolution of SSF. This method is an efficient way to greatly reduce the number of simulated particles and at the same time to sharpen the final field. For confirmation, we also ran the reference case simulation focusing on the Daoulas bay, for which we set the initial number of particles per mesh cell to 200,400, 800 and 1600 to reach the computation limit of the supercomputer. The local coefficient estimated using different numbers of particles remained unchanged due to the overlap in the initial cloud technique. The maximum coefficient estimation was found geometrically by setting OD equal to SSF. It was also possible to sample the tidal signal with 13 simulations, which granted independence from the launching date and also increased the final number of estimations. For example, in the reference case, the median estimate per cell inside the bay was done over 210 trajectory centroids (minimum 13 and maximum 5800). This is a key element for successfully mapping diffusivities across the entire bay. The minimum redundancy was observed close to the coastline in shallow areas. 
To obtain a coefficient that has statistical representativeness, the DES radius must be chosen so that there are enough particles within each cloud to estimate a variance at the initial time (typically $\mathrm{N}>=100$ ). This constraint can be translated as the initial distance between particles (SSF) being much lower than the DES. The DES may have a strong impact on the final coefficient. Richardson (1926) highlighted in an atmospheric study that the dispersion rate under assumptions increased with the initial cloud size according to the $4 / 3$ power law. Okubo (1971) related the dispersion rate to a 1.15 power law. This indicates that the relative dispersion is scale-dependent (LaCasce 2008). We estimated the local diffusivity for the mean tide condition with different values of DES (Fig. 8). To compare the radius effect only, we chose diverse hydrodynamic places in the bay and scaled down the coefficient by averaging the value over a $250 \mathrm{~m}$ square. The relative difference compared with a $50 \mathrm{~m}$ DES was then plotted. This plot highlights the lack of agreement with Richardson's law. It is even lower than the power of 1.15 found by Okubo (1971) based on dye-release experiments in the open sea. More recently, Soomere et al. (2011) demonstrated, based on observations in a large estuary, that different spatial scales may follow radically different laws for relative dispersion. We clearly see different impacts according to the bay under consideration, the major difference being in the place where the local diffusivity was less than $1 \mathrm{~m}^{2} . \mathrm{s}^{-1}$, and amplified by a factor of 3 with a $250 \mathrm{~m}$ DES. In contrast, in the Goulet and in the centre of the bay where the values were the highest (greater than $100 \mathrm{~m}^{2} . \mathrm{s}^{-1}$ ), the dispersion was not affected by an increase in the DES value. Therefore, it is hard to find a relationship that covers all situations. This variability is due to the complex combination of advection and diffusion processes in this type of anisotropic area. One last comment can be made on choosing a scanning radius smaller than the hydrodynamic model resolution: although it is numerically possible, the captured dispersion is mainly due to the numerical diffusion due to the advection scheme and does not represent a pure physical event. We used the same hydrodynamic model by decreasing the horizontal resolution to $250 \mathrm{~m}$. The local diffusivities estimated with a $250 \mathrm{~m}$ DES were also of the same order of magnitude. Of course, local structures were not similar due to the loss of resolution and bathymetric smoothing. This similarity is definitely encouraging for comparisons between different geographic bays.

\section{Fig.8}

The horizontal resolution of the final grid is intrinsically correlated with the OD and SSF to obtain sufficient numbers of cloud barycentres within each final mesh cell. This latter scale will depend on the application and the geographic extent. The classic approach tends to take the same grid as the hydrodynamic model in environmental studies, except in the case of an inter-comparison with another geographic area, for which it is recommended to take the highest hydrodynamic resolution of both models.

\section{Impact of tide}

To identify the impact of the tidal regime on this assessment of dispersion, we also tested mean neap and mean spring tide flows without any atmospheric forcings. The results are shown in Fig. 9a (neap tide) and 9b (spring tide). They must be compared with the reference case (Fig. 4a). As expected, the tidal conditions strongly affect the whole domain (sheltered or exposed areas). The diffusivity was an order of magnitude higher during spring tides (that is to say a factor 10) than during neap tides, regardless of the area considered. The increase in the advection magnitude between neap and spring tidal cycles and therefore the resulting tidal excursions -- one of the main drivers of dispersion -- may heuristically explain this result. During neap tides, barotropic currents never exceeded $2 \mathrm{~m} \cdot \mathrm{s}^{-1}$ in the bay, whereas they 
reached $4 \mathrm{~m} . \mathrm{s}^{-1}$ during spring tides. Nonetheless, interestingly, the global structure remained the same. There is still a clear separation between the centre of the bay, the eastern parts and the sheltered coves. The spring tide condition enhanced the areas with rather low dispersion capabilities (i.e. below $10 \mathrm{~m}^{2} . \mathrm{s}^{-1}$ ) over a tidal cycle. These small areas are at the far ends of the coves (Fret, Roscanvel and the military harbour) and appear to be the most isolated places within the Bay of Brest because they are weakly dispersive regardless of the tidal regime.

The anisotropic coefficients (not shown) linked to these dispersive fields (Fig. 9a and 9b) are barely sensitive to the tidal regime. They remained similar with a ratio (between the major and minor axes of dispersion) greater than 2 everywhere in the bay for flattened ellipses. This result is due in both cases to the horizontal advection responsible for the lengthening of the tidal excursion driven by bathymetric shapes.

\section{Fig. 9 a, b}

Finally, one must keep in mind that the real situation is a succession of different tidal regimes, shifting perpetually from neap to spring conditions, so as that the dispersion results in a combination of the situations described in Fig. 9 and Fig 4.a, which are highly non-linear and hard to predict. This observation must also be combined with the fact that for longer time lags (i.e. more than a one tidal cycle), stationarity was not reached, as mentioned above.

\section{Impact of meteorological forcings}

The question addressed here was to identify the role played by the meteorological forcings with respect to tide, and especially to determine the dominant process. The meteorological forcing adds both horizontal transport and vertical mixing, which are closely related and probably interact with the main dominant flow in a non-linear manner. Moreover, the understanding of the meteorological effects on such global indicators may be an exhausting task, because its variability at mid-latitudes is difficult to reduce and the events combine in a complex and non-linear way along coasts.

For illustrative purposes, we focused on the two dominant regimes extracted from the analysis of a long-term series (south-westerly and north-easterly winds), whose intensities have been reduced to two cases (light $5 \mathrm{~m} . \mathrm{s}^{-1}$ and moderate $10 \mathrm{~m} . \mathrm{s}^{-1}$ wind). Moreover, although these experiments (maintaining homogeneous wind over the entire bay and constant over one tidal cycle, the length of the experiment) are rather simplistic, they provide a rough idea of the relative weight of winds and tides over the dispersion processes.

First, all the scenarios on the dispersive fields (Fig. 10 a, c, e, g) still exhibited marked structures, which remain more or less the same as those obtained in the reference case (Fig. 4a): the sheltered areas are still set apart. Overall, as expected intuitively, the dispersion over the bay was enhanced. It is obvious for moderate winds conditions (Fig. 10c and $10 \mathrm{~g}$ ) and perhaps a little less evident under light wind conditions (Fig. 10a and 10e). Second, the wind intensity tends to blur the overall picture with respect to the same scenario at weaker intensity; it causes the overall net enhancement of dispersion.

In either case, there was no preferential dispersion direction and the anisotropic coefficient remained high (above 2 everywhere in the bay). The direction of the fastest spread did not change with the direction of the wind. In Fig. 10, the wind impact is not uniform geographically and has a rather complex structure: there is no clear signal in the centre of the bay, where dispersion remains generally almost unchanged. This structure is due to the combination of strong 
advection that occurs during the ebb and flood of each tide and large depth rendering the flow less sensitive to the wind effects, i.e. additional transport or turbulence.

In contrast, as soon as the depth decreased, significant differences appeared. In light wind conditions, the relative difference fields are more confusing, either under south-westerly or north-easterly winds. It is even hard to describe, apart from the relative stability of the central pool; however, the far ends of the coves are the most affected (Fret, Roscanvel, etc.) and the diffusivity was enhanced at least by a factor of 3 .

An intriguing point is the large decrease in the dispersion coherent area observable in the northern part of the bay under both light wind conditions (i.e. south-westerly and north-easterly winds). This area is more stressed under south-westerly winds, during which a large negative patch appears over the Keraliou bed, as if the winds had induced a confinement of the particle clouds.

In addition, the modifications are noisy with alternating hot spots of positive and negative changes, which seem almost randomly organised on the scale of a few kilometres. Under rougher wind conditions, the local diffusivity increased nearly everywhere except in the main deep pool, where the dispersion remained stable and around the Keraliou bed in shallow areas where it decreased. In the Roscanvel or Fret coves and next to Lomergat, the coefficient increased slightly less with the north-easterly wind. The effect is the opposite for the Daoulas Cove with the south-westerly wind. Theses local differences, located in the direction of the wind, can be partially explained by the particles that are either pushed away or maintained in their initial area.

\section{Fig. 10 a, b, c, d, e, f, g, h}

\section{Discussion}

The present study sought to derive a local diffusivity as a robust indicator of dispersion in semi-enclosed macrotidal coastal areas. It attempted to deal with the large advection process (more than $1 \mathrm{~m} . \mathrm{s}^{-1}$ during mid-tide conditions) associated with very large tidal excursions, even at the residual scale, which peak at $10 \mathrm{~cm} \cdot \mathrm{s}^{-1}$ (see Fig. $3 \mathrm{~b}$ ). This coefficient relies on the barycentric repositioning method to filter out the tidal scale and give coherence to the computed fields. The second need was to provide a local indication of dispersion at relatively short time scales (a few tidal cycles). The framework used relies on the theoretical definition of relative dispersion and diffusivity (e.g. LaCasce 2008). It can be used, with simplicity, in several applications from biological processes (larval dispersion, pathogen contagion) to environmental effects (micro-plastic concentration, sedimentation nature).

The depictions resulted in fields with coherent structures (Fig. 4a), which can be linked to macro-descriptors of flow (such as tidal excursion or vertical diffusion) in a more or less straightforward way: none of the variables selected to explain the diffusivity structure give a simple relationship, which -- as expected -- given the many flow regimes (from weak and highly sensitive to the wind to strong currents relatively insensitive to meteorological forcings, forming channelled flows in narrow estuaries and weakly constrained by the coastline) and local basin geometry occur and assemble in a numerous combinations.

Moreover, the revealed structures are robust with respect to the main drivers of the hydrodynamic regimes (tides and meteorological forcings). These structures are thus relatively realistic, given that they are expected to be almost always 
present regardless of the sequence of events. Additionally, the indicator clearly illustrates the relative contribution of the various processes to diffusivity. Here, the tidal effects overwhelm the meteorological forcings even though the latter can modulate tidal diffusivity.

The estimation depends on the time scale of the tide cycle because the Bay of Brest is macrotidal with a strong semidiurnal signal. This time constraint is an important counterparty for the delicate choice of a control volume. The time integrating period in a microtidal or non-tidal environments is not well defined. As we saw, the structure of the coefficient is reliable over several tide cycles, thus it can be likely applied to microtidal areas with diurnal or semidiurnal tides. The repositioning technique, which reduces bias, was established based on the presence of residual circulation. In a closed lagoon, the usefulness of the repositioning technique with the cloud barycentre after a defined time (12, 24 hours) must be determined.

This dependency is a limitation of the dispersive indicator whose field structure tends to become less precise over time. We did not explore time scales longer than four tidal cycles. At longer scales, barycentric positioning experiences a dramatic loss of precision (because the net residual displacements are too large), and this method generates imprecise depictions. The overall picture is not only imprecise, but also false given that, with such large displacements, the dispersion measured is that experienced over a wide area, making it difficult to position it precisely within the basin. These inherent geographic limitations caused by the repositioning technique are typically visible near the Goulet or adjacent to the dike of the Brest harbour. There is a clear deformation of the final field due to the particularity of the cloud trajectory shapes over one cycle, which are not elliptic. It also becomes difficult to apply this technique directly in tortuous or curved estuaries by keeping the land mask, because the barycentres of many tracks are located on land. However, the final distortion due to the coastline is not a binding constraint to unravel the final diffusivity.

At longer time scales (i.e. more than a few tidal cycles), it becomes harder to separate advection from diffusivity because they are intricately linked. Thus, obtaining a spatialised generic (or intrinsic) picture of the bay at longer time scales is not only hardly possible, but also likely to be meaningless. Any additional effort would be useless, and should be problem-oriented, i.e. dedicated to a particular situation. This kind of approach includes those previously cited, such as like all the time scale previously cited like transit, exposure, flushing that would shed light on additional aspects and should be combined.

Here we use a Lagrangian approach with a large number of particles (100 million particle trajectories simulated in 13 runs to provide one situation) that is manageable only with the latest available computational power. A single simulation (here, 13 simulations, corresponding to each hour of the tidal clock) makes it possible to aggregate a posteriori the particles at different spatial scales and at different resolutions or even different manners to: (1) provide properly and fully filled fields with a low level of noise by multiplying the number of samples (e.g. by using sliding and overlapping clouds), (2) investigate dispersion at various spatial scales and (3) investigate some methodological aspects, such as convergence, with respect to the resolution.

A Eulerian method could have been used for the dispersion estimation. With respect to numerical time integrating constraint, the spreading result would have been the same (van Sebille et al. 2018). In terms of computation means however, the Lagrangian approach is clearly superior to the Eulerian one. In an Eulerian approach, we would have simulated one tracer for every hydrodynamic mesh cell which is not possible for such a large area, although this method 
has been recently used for connectivity purposes by compiling several meshes at larger horizontal scales (Ménesguen et al. 2018). Both methods allowed to combine transport capability and dispersion at longer time scales and provide a quantitative link between two given points (e.g. Jacobi and Jonsson 2011) to understand coastal spreading scenarios. Eventually, this method is particularly relevant for getting local diffusivity estimation as much as possible everywhere over the domain under study.

The change in dispersion over a tidal cycle makes it possible to deduce diffusivity, which is realistic as long as there is stationary behaviour over a temporal window. The relative dispersion and the diffusivity deduced may be at least theoretically related to the turbulence regime (Foussard et al. 2017) in some particular cases (quasi-geostrophic or surface quasi-geostrophic turbulence). We are here far beyond the scope of these kind of regimes, because it is difficult to identify the kinds of turbulence occurring; in the particular situation studied, the flow is highly ageostrophic, nonstationary and heterogeneous. Therefore, we are likely to obtain different coexisting turbulence regimes. These disparities make comparisons with theoretical or empirical results (power law of Richardson 1926 or Okubo 1971) rather difficult and even may be not realistic.

Despite a quite large number of particles introduced, the initial distance between particles (SSF scale) is an additional cut-off frequency to the one introduced by the grid cell size of the model. This has not been investigated nor introduced (e.g. through a random walk during the particle tracking). The DES radius scale is also a crucial aspect for the estimation of this indicator's intensity. In fact, the optimal resolution is mainly determined on the basis of numerical considerations (computing time, available memory, file size, etc.). The manner of simulating at certain scales leads to an unavoidable frequency cut-off for diffusion processes. The model's ability to properly capture the small scales mainly responsible for the dispersion is an ongoing topic of debate. One relevant illustration of this occurs within the lateral boundary layer of the Goulet de Brest where -- at some tidal phases -- small eddies grew behind points. However, the relative diffusivity at this tidal time scale is much higher in terms of orders of magnitude in complex tidal areas than those small scales. In very sheltered environments, this may be problematic, but here we are more concerned with discerning local patterns than with distinguishing them. The DES scale nevertheless incorporates all these physical terms and is the major parameter to choose to compare several geographic places with different hydrodynamic resolution models.

\section{Conclusion}

The local diffusivity exhibited here is a new robust indicator whose structure persists under different hydrodynamic and meteorological forcings. It gives information directly about areas with low dispersion capacities. It is a powerful indicator that provides a short-term clarification a priori over a geographic area, although limited by advection time scales. This is partially overcome using a barycentre repositioning technique that plays a large role in increasing the consistency of the final field.

This indicator thus helps understand the combined effect of advection and diffusion. The coefficient values are qualitative and meaningful to understand realistic dispersion patch. The general method is easily applicable to other environmental coastal areas where hydrodynamic models exist. It will help to define a degree of differentiation between specific areas of interest at short time scales which is a frequent request of biologists and policy makers. The local diffusivity is an additional tool that supplements the whole set of previously known indicators that have a volume- 
dependency constraint. This coefficient provides guidance for understanding the fate of the release of material (e.g. pollutants, waste discharges, larval dispersion) and unavoidable concentration fluctuations in such systems. This methodology can potentially combine or integrate the long-term renewal time scales to evaluate the risk of water pollution (Grifoll et al. 2013; Cucco and Umgiesser 2015). It is now worth testing in other coastal ecosystems of interest. 


\section{References}

Andutta FP, Helfer F, de Miranda LB, et al (2016) An assessment of transport timescales and return coefficient in adjacent tropical estuaries. Cont Shelf Res 124:49-62. doi: 10.1016/J.CSR.2016.05.006

Auffret G-A (1983) Dynamique sédimentaire de la marge continentale celtique - Evolution Cénozoïque - Spécificité du Pleistocène supérieur et de l'Holocène. Université de Bordeaux I

Bailly du Bois P, Dumas F (2005) Fast hydrodynamic model for medium- and long-term dispersion in seawater in the English Channel and southern North Sea, qualitative and quantitative validation by radionuclide tracers. Ocean Model 9:169-210. doi: 10.1016/j.ocemod.2004.07.004

Bendtsen J, Gustafsson KE, Söderkvist J, Hansen JLS (2009) Ventilation of bottom water in the North Sea-Baltic Sea transition zone. J Mar Syst 75:138-149. doi: 10.1016/J.JMARSYS.2008.08.006

Blumberg AF, Mellor GL (1987) A Description of a Three-Dimensional Coastal Ocean Circulation Model. ThreeDimensional Coast. Ocean Model.

Bolin B, Rodhe H (1973) A note on the concepts of age distribution and transit time in natural reservoirs. Tellus 25:5862. doi: 10.1111/j.2153-3490.1973.tb01594.x

Brown J, MacMahan J, Reniers A, Thornton E (2009) Surf zone diffusivity on a rip-channeled beach. J Geophys Res 114:C11015. doi: 10.1029/2008JC005158

Capet X, Campos EJ, Paiva AM (2008) Submesoscale activity over the Argentinian shelf. Geophys Res Lett 35:2-6. doi: 10.1029/2008GL034736

Charria G, Lazure P, Le Cann B, et al (2013) Surface layer circulation derived from Lagrangian drifters in the Bay of Biscay. J Mar Syst 109-110:S60-S76. doi: 10.1016/j.jmarsys.2011.09.015

Cucco A, Umgiesser G (2015) The Trapping Index : How to integrate the Eulerian and the Lagrangian approach for the computation of the transport time scales of semi-enclosed basins. Mar Pollut Bull 98:210-220. doi: 10.1016/j.marpolbul.2015.06.048

de Brauwere A, de Brye B, Blaise S, Deleersnijder E (2011) Residence time, exposure time and connectivity in the Scheldt Estuary. J Mar Syst 84:85-95. doi: 10.1016/j.jmarsys.2010.10.001

de Brye B, de Brauwere A, Gourgue O, et al (2012) Water renewal timescales in the Scheldt Estuary. J Mar Syst 94:7486. doi: 10.1016/J.JMARSYS.2011.10.013

De Kok JM (1994) Tidal averaging and models for anisotropic dispersion in coastal waters. Tellus A 46:160-177

Deleersnijder E, Campin JM, Delhez EJM (2001) The concept of age in marine modelling I. Theory and preliminary model results. J Mar Syst 28:229-267. doi: 10.1016/S0924-7963(01)00026-4

Delhez EJM (2006) Transient residence and exposure times. Ocean Sci 2:1-9. doi: 10.5194/os-2-1-2006

Delhez EJM (1996) On the residual advection of passive constituents. J Mar Syst 8:147-169. doi: 10.1016/09247963(96)00004-8 
Delhez ÉJM, de Brye B, de Brauwere A, Deleersnijder É (2014) Residence time vs influence time. J Mar Syst 132:185195. doi: 10.1016/J.JMARSYS.2013.12.005

Delhez ÉJM, Heemink AW, Deleersnijder É (2004) Residence time in a semi-enclosed domain from the solution of an adjoint problem. Estuar Coast Shelf Sci 61:691-702. doi: 10.1016/j.ecss.2004.07.013

Döös K, Rupolo V, Brodeau L (2011) Dispersion of surface drifters and model-simulated trajectories. Ocean Model 39:301-310. doi: 10.1016/J.OCEMOD.2011.05.005

Fiandrino A, Ouisse V, Dumas F, et al (2017) Spatial patterns in coastal lagoons related to the hydrodynamics of seawater intrusion. Mar Pollut Bull 119:132-144. doi: 10.1016/j.marpolbul.2017.03.006

Foussard A, Berti S, Perrot X, Lapeyre G (2017) Relative dispersion in generalized two-dimensional turbulence. J Fluid Mech 821:358-383. doi: 10.1017/jfm.2017.253

Gräwe U, Deleersnijder E, Ali Muttaqi Shah SH, Heemink AW (2012) Why the Euler scheme in particle tracking is not enough: The shallow-sea pycnocline test case. Ocean Dyn 62:501-514. doi: 10.1007/s10236-012-0523-y

Gregoire G, Ehrhold A, Le Roy P, et al (2016) Modern morpho-sedimentological patterns in a tide-dominated estuary system: the Bay of Brest (west Britanny, France). J Maps 12:1152-1159. doi: 10.1080/17445647.2016.1139514

Grifoll M, Del Campo A, Espino M, et al (2013) Water renewal and risk assessment of water pollution in semi-enclosed domains: Application to Bilbao Harbour (Bay of Biscay). J Mar Syst 109-110:S241-S251. doi: 10.1016/j.jmarsys.2011.07.010

Guillou N (2007) Effects of the heterogeneity of bottom sediments and wave-current interaction on subtidal hydrodynamics and sediment transport - applications to the eastern English Channel and off Brittany. Université de Bretagne occidentale - Brest

Haza AC, Poje AC, Özgökmen TM, Martin P (2008) Relative dispersion from a high-resolution coastal model of the Adriatic Sea. Ocean Model 22:48-65. doi: 10.1016/j.ocemod.2008.01.006

Jacobi MN, Jonsson PR (2011) Optimal networks of nature reserves can be found through eigenvalue perturbation theory of the connectivity matrix. Ecol Appl 21:1861-1870. doi: 10.1890/10-0915.1

Jo YB, Park S-H, Choi HY, et al (2019) SOPHIA: Development of Lagrangian-based CFD code for nuclear thermalhydraulics and safety applications. Ann Nucl Energy 124:132-149. doi: 10.1016/j.anucene.2018.09.005

Kamenkovich I, Berloff P, Pedlosky J (2009) Anisotropic Material Transport by Eddies and Eddy-Driven Currents in a Model of the North Atlantic. J Phys Oceanogr 39:3162-3175. doi: 10.1175/2009JPO4239.1

Kininmonth SJ, De'ath G, Possingham HP (2010) Graph theoretic topology of the Great but small Barrier Reef world. Theor Ecol 3:75-88. doi: 10.1007/s12080-009-0055-3

Kool JT, Moilanen A, Treml EA (2013) Population connectivity: recent advances and new perspectives. Landsc Ecol 28:165-185. doi: 10.1007/s10980-012-9819-z

LaCasce JH (2008) Statistics from Lagrangian observations. Prog Oceanogr 77:1-29. doi: 10.1016/j.pocean.2008.02.002 
LaCasce JH, Ohlmann C (2003) Relative dispersion at the surface of the Gulf of Mexico. J Mar Res 61:285-312. doi: $10.1357 / 002224003322201205$

Lazure P, Dumas F (2008) An external-internal mode coupling for a 3D hydrodynamical model for applications at regional scale (MARS). Adv Water Resour 31:233-250. doi: 10.1016/J.ADVWATRES.2007.06.010

Lazure P, Dumas F, Vrignaud C (2008) Circulation on the Armorican shelf (Bay of Biscay) in autumn. J Mar Syst 72:218-237. doi: 10.1016/J.JMARSYS.2007.09.011

Le Roy R, Simon B (2003) Réalisation et validation d'un modèle de marée en Manche et dans le Golfe de Gascogne. Application à la réalisation d'un nouveau programme de réduction des sondages bathymétriques

Liu G, Liu Z, Gao H, et al (2012) Simulation of the Lagrangian tide-induced residual velocity in a tide-dominated coastal system: a case study of Jiaozhou Bay, China. Ocean Dyn 62:1443-1456. doi: 10.1007/s10236-012-0577-x

Meier HEM (2005) Modeling the age of Baltic Seawater masses: Quantification and steady state sensitivity experiments. J Geophys Res Ocean 110:. doi: 10.1029/2004JC002607

Ménesguen A, Hachet A, Grégoris T (2018) Modelling benthic invasion by the colonial gastropod Crepidula fornicata and its competition with the bivalve Pecten maximus. 2. Coupling the $0 \mathrm{D}$ model of colony-forming species to a connectivity matrix for a realistic distributed simulation of benthic invasion. Ecol Modell 375:30-44. doi: 10.1016/J.ECOLMODEL.2018.02.015

Monsen NE, Cloern JE, Lucas L V, Monismith SG (2002) A comment on the use of flushing time, residence time, and age as transport time scales. Limnol Oceanogr 47:1545-1553. doi: 10.4319/1o.2002.47.5.1545

Mouchet A, Cornaton F, Deleersnijder É, M. Delhez ÉJ (2016) Partial ages: diagnosing transport processes by means of multiple clocks. Ocean Dyn 66:367-386. doi: 10.1007/s10236-016-0922-6

Muller H, Blanke B, Dumas F, et al (2009) Estimating the Lagrangian residual circulation in the Iroise Sea. J Mar Syst 78:S17-S36. doi: 10.1016/J.JMARSYS.2009.01.008

Nihoul JCJ, Ronday FC (1975) The influence of the "tidal stress" on the residual circulation. Tellus 27:484-490. doi: 10.1111/j.2153-3490.1975.tb01701.x

North EW, Hood RR, Chao S, Sanford LP (2006) Using a random displacement model to simulate turbulent particle motion in a baroclinic frontal zone: A new implementation scheme and model performance tests. 60:365-380. doi: 10.1016/j.jmarsys.2005.08.003

O'Dwyer J, Williams RG, LaCasce JH, Speer KG (2000) Does the Potential Vorticity Distribution Constrain the Spreading of Floats in the North Atlantic? J Phys Oceanogr 30:721-732. doi: 10.1175/15200485(2000)030<0721:DTPVDC>2.0.CO;2

Okubo A (1971) Oceanic diffusion diagrams. Deep Res Oceanogr Abstr 18:789-802. doi: 10.1016/00117471(71)90046-5

Pape O Le, Menesguen A (1997) Hydrodynamic prevention of eutrophication in the Bay of Brest (France), a modelling approach. J Mar Syst 12:171-186. doi: 10.1016/S0924-7963(96)00096-6 
Petton S (2010) Etude des processus hydrodynamiques et hydro-sédimentaires affectant un estran de type marais salé de la rade de Brest (anse de Penfoul) colonisé par l'espèce invasive spartine (Spartina alterniflora Loisel). Université de Bretagne Occidentale.

Petton S, David LB, Haurie A, Pouvreau S (2016) HOMER Campaign: Mooring time series. SEANOE. https://doi.org/10.17882/43082

Petton S, Le Roy V, Bellec G, et al (2018) Marine environmental station database of Daoulas bay. SENONE. https://doi.org/10.17882/42493

Pineda J, Hare J, Sponaugle S (2007) Larval Transport and Dispersal in the Coastal Ocean and Consequences for Population Connectivity. Oceanography 20:22-39. doi: 10.5670/oceanog.2007.27

Plus M, Dumas F, Stanisière J-Y, Maurer D (2009) Hydrodynamic characterization of the Arcachon Bay, using modelderived descriptors. Cont Shelf Res 29:1008-1013. doi: 10.1016/J.CSR.2008.12.016

Richardson LF (1926) Atmospheric Diffusion Shown on a Distance-Neighbour Graph. Proc R Soc A Math Phys Eng Sci 110:709-737. doi: 10.1098/rspa.1926.0043

Ridderinkhof H, Loder JW (1994) Lagrangian Characterization of Circulation over Submarine Banks with Application to the Outer Gulf of Maine. J Phys Oceanogr 24:1184-1200. doi: 10.1175/15200485(1994)024<1184:LCOCOS>2.0.CO;2

Ridderinkhof H, Zimmerman JTF (1992) Chaotic Stirring in a Tidal System. Science (80- ) 258:1107 LP - 1111. doi: $10.1126 /$ science.258.5085.1107

Rossi V, Ser-Giacomi E, López C, Hernández-García E (2014) Hydrodynamic provinces and oceanic connectivity from a transport network help designing marine reserves. Geophys Res Lett 41:2883-2891. doi: 10.1002/2014GL059540

Rypina II, Kamenkovich I, Berloff P, Pratt LJ (2012) Eddy-Induced Particle Dispersion in the Near-Surface North Atlantic. J Phys Oceanogr 42:2206-2228. doi: 10.1175/JPO-D-11-0191.1

Rypina II, Kirincich A, Lentz S, Sundermeyer M (2016) Investigating the Eddy Diffusivity Concept in the Coastal Ocean. J Phys Oceanogr 46:2201-2218. doi: 10.1175/JPO-D-16-0020.1

Salomon JC, Breton M (1991) Numerical study of the dispersive capacity of the bay of Brest, France towards dissolved substances. Environ. Hydraul. 459-464

Salomon JC, Garreau P, Breton M (1996) The Lagrangian Barycentric method to compute 2D and 3D long term dispersion in tidal environments. In: Mixing in Estuaries and Coastal Seas. pp 59-76

Sawford B (2001) Turbulent Relative Dispersion. Annu Rev Fluid Mech 33:289-317. doi: 10.1146/annurev.fluid.33.1.289

Shah SHAM, Heemink AW, Gräwe U, Deleersnijder E (2013) Adaptive time stepping algorithm for Lagrangian transport models: Theory and idealised test cases. Ocean Model 68:9-21. doi: 10.1016/j.ocemod.2013.04.001

Simpson JH, Hunter JR (1974) Fronts in the Irish Sea. Nature 250:404-406. doi: 10.1038/250404a0 
Soomere T, Viidebaum M, Kalda J (2011) On dispersion properties of surface motions in the Gulf of Finland. Proc Est Acad Sci 60:269. doi: 10.3176/proc.2011.4.07

Suara K, Chanson H, Borgas M, Brown RJ (2017) Relative dispersion of clustered drifters in a small micro-tidal estuary. Estuar Coast Shelf Sci 194:1-15. doi: 10.1016/j.ecss.2017.05.001

Thomas CJ, Lambrechts J, Wolanski E, et al (2014) Numerical modelling and graph theory tools to study ecological connectivity in the Great Barrier Reef. Ecol Modell 272:160-174. doi: 10.1016/J.ECOLMODEL.2013.10.002

Umlauf L, Burchard H (2003) A generic length-scale equation for geophysical turbulence models. J Mar Res 61:235265. doi: $10.1357 / 002224003322005087$

van Sebille E, Griffies SM, Abernathey R, et al (2018) Lagrangian ocean analysis: Fundamentals and practices. Ocean Model. 121:49-75

Viero D Pietro, Defina A (2016) Renewal time scales in tidal basins: Climbing the Tower of Babel. Sustain Hydraul Era Glob Chang 338-345

Visser A (1997) Using random walk models to simulate the vertical distribution of particles in a turbulent water column. Mar Ecol Prog Ser 158:275-281. doi: 10.3354/meps 158275

Warner JC, Sherwood CR, Arango HG, Signell RP (2005) Performance of four turbulence closure models implemented using a generic length scale method. Ocean Model 8:81-113. doi: 10.1016/j.ocemod.2003.12.003

Wei H, Hainbucher D, Pohlmann T, et al (2004) Tidal-induced Lagrangian and Eulerian mean circulation in the Bohai Sea. J Mar Syst 44:141-151. doi: 10.1016/J.JMARSYS.2003.09.007

White L, Deleersnijder E (2007) Diagnoses of vertical transport in a three-dimensional finite element model of the tidal circulation around an island. Estuar Coast Shelf Sci 74:655-669. doi: 10.1016/j.ecss.2006.07.014

Zimmerman JTF (1976) Mixing and flushing of tidal embayments in the western Dutch Wadden Sea part I: Distribution of salinity and calculation of mixing time scales. Netherlands J. Sea Res. 10:149-191 


\section{List of Figures}

Fig. 1 (a) Example of the dispersion of a patch over a tidal cycle. The green star represents the trajectories' barycentre. The blue arrows are the major axis, denoted by $\xi$, and the minor axis, denoted by $\eta$, of the ellipse's dispersion patch. (b) Construction of the patches (in blue circles) of a given radius (DES) with the overlap distance (OD). The radius is equal to the hydrodynamic resolution $(d x)$.
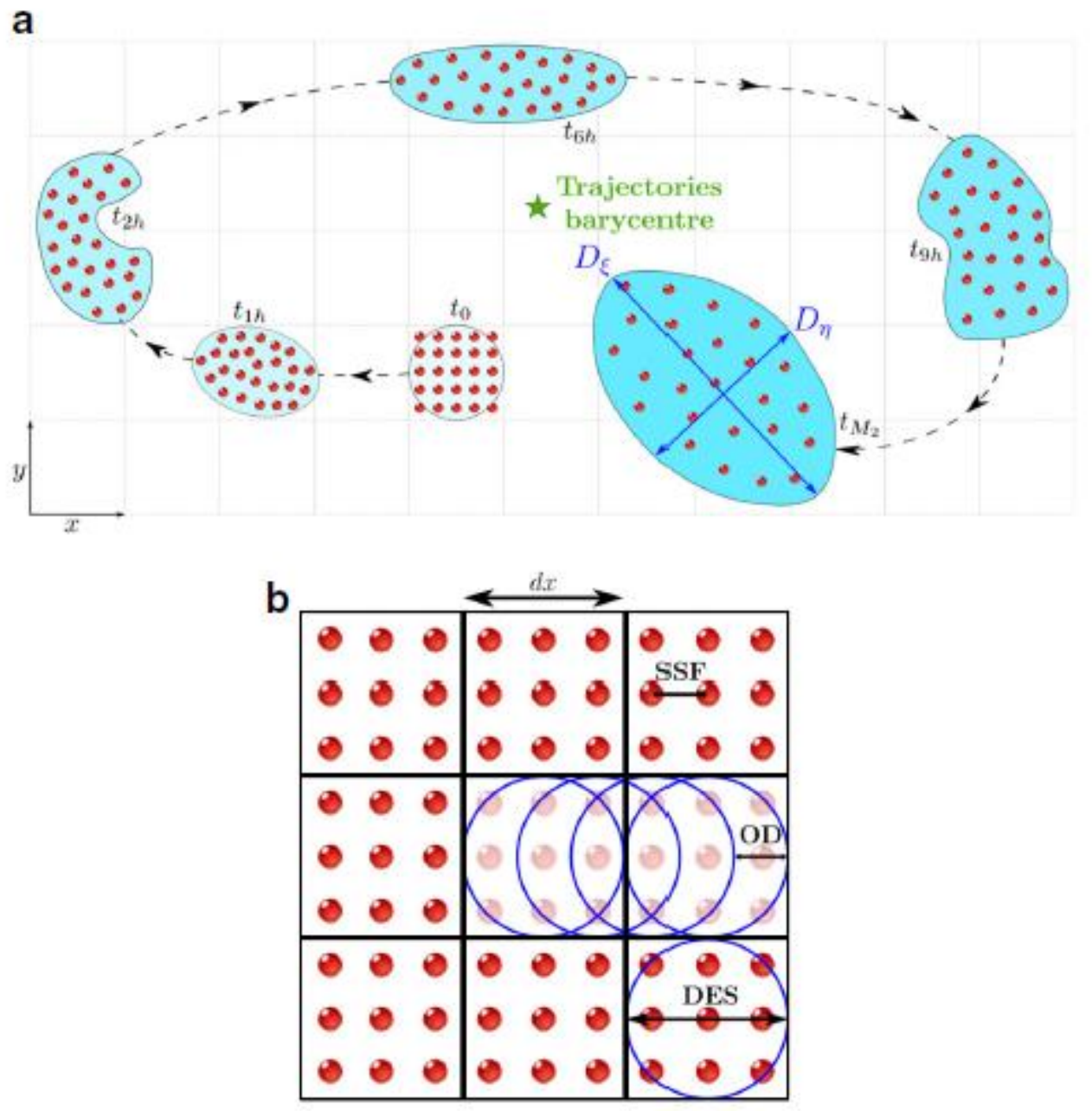
Fig. 2 Bathymetry of the Bay of Brest (Source: Ifremer / SHOM). The solid red line represents the geographic extent of the hydrodynamic 3D model. The dashed red line indicates for the coarser 2D model used for propagating the tide signal.

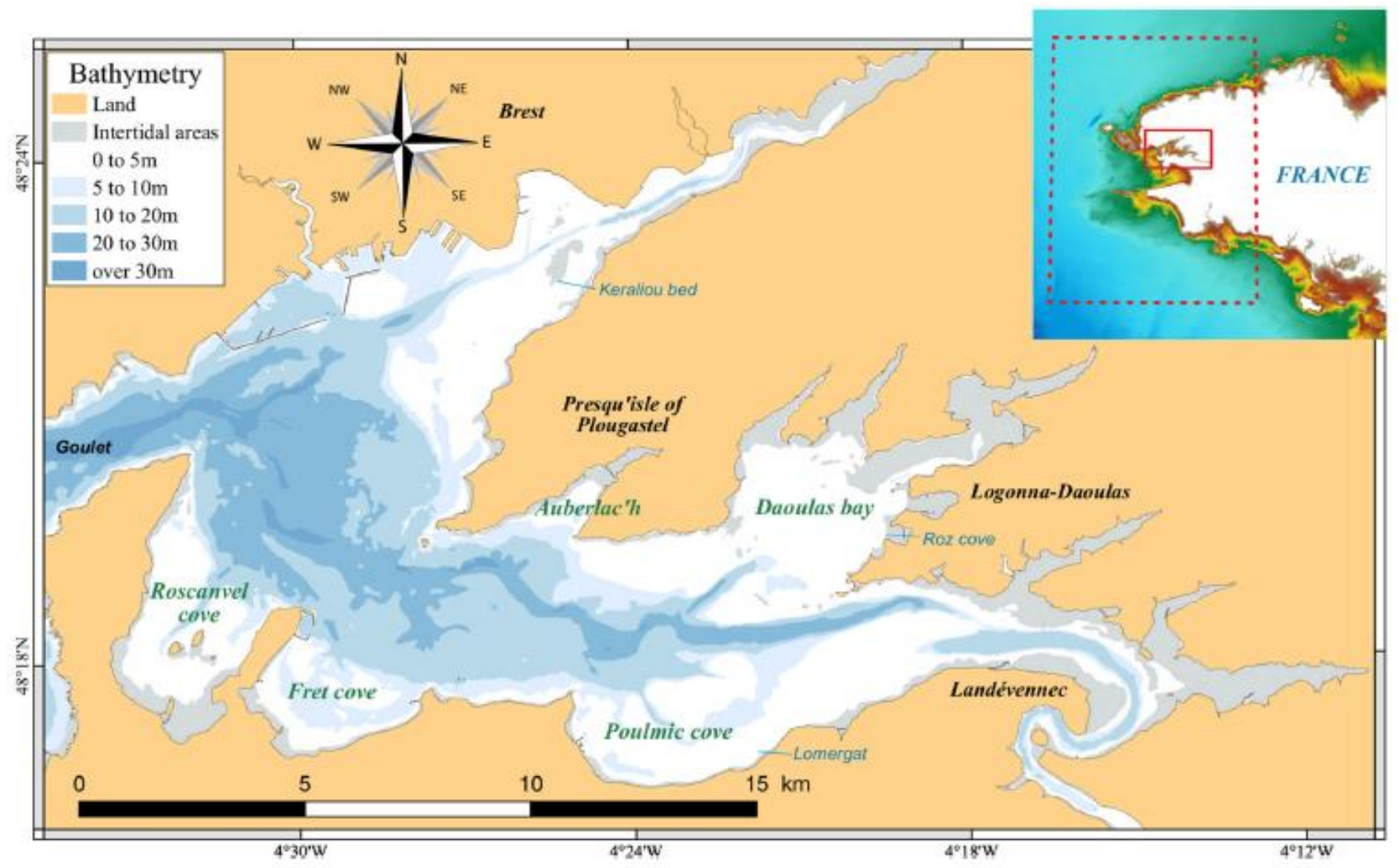


Fig. 3 Lagrangian tidal residual current fields without barycentric repositioning (a) and with barycentric repositioning (b) for mean tide conditions without any meteorological forcing.

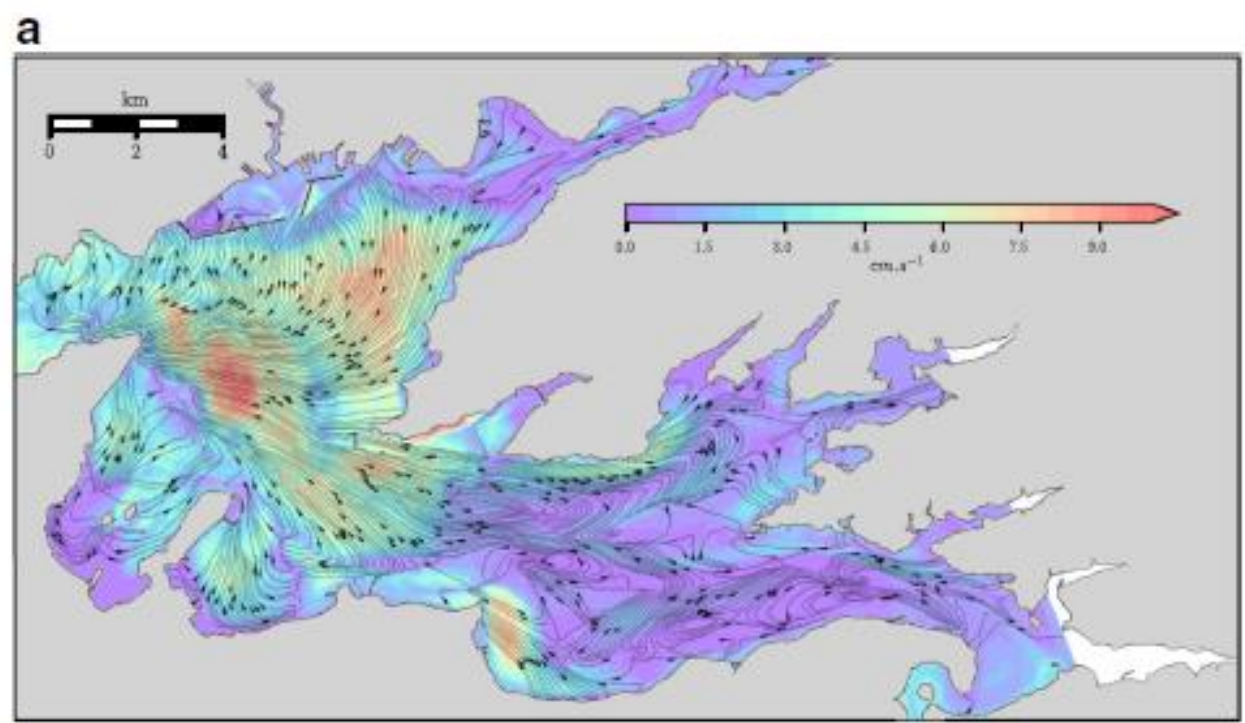

b

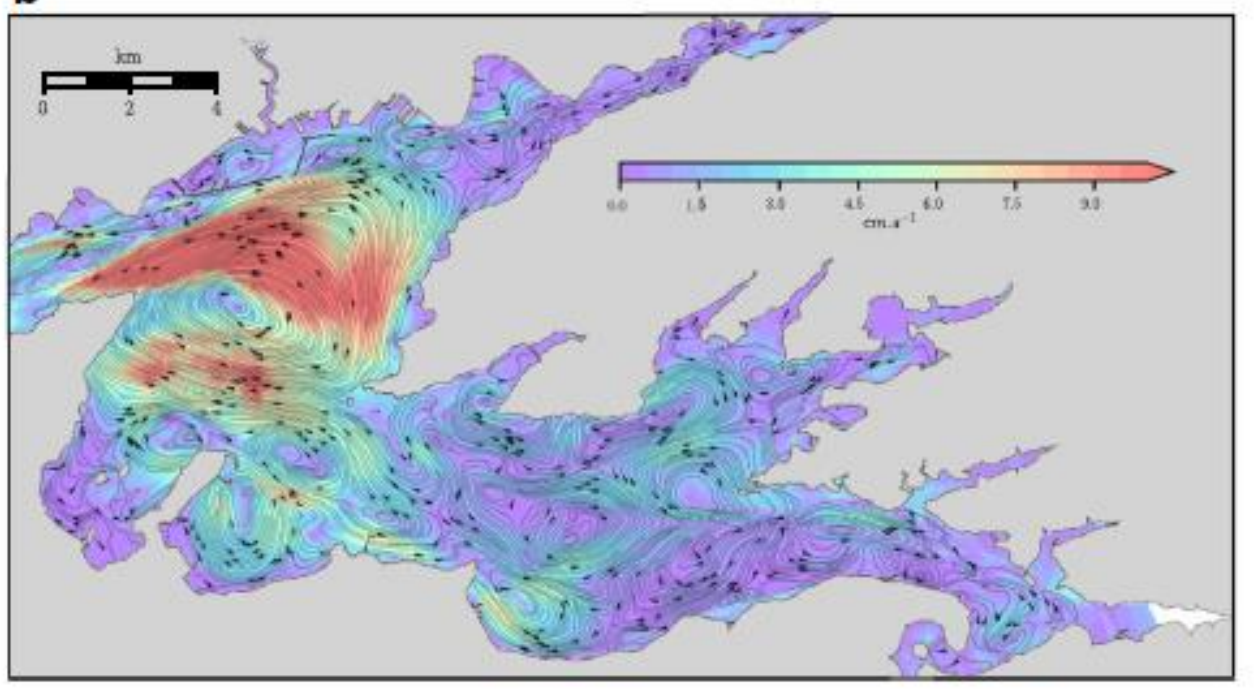


Fig. 4 (a) Local diffusivity $K_{\xi}$ estimation and (b) anisotropic coefficient $R_{D}$ over a mean tidal cycle with barycentric repositioning without any meteorological forcing.

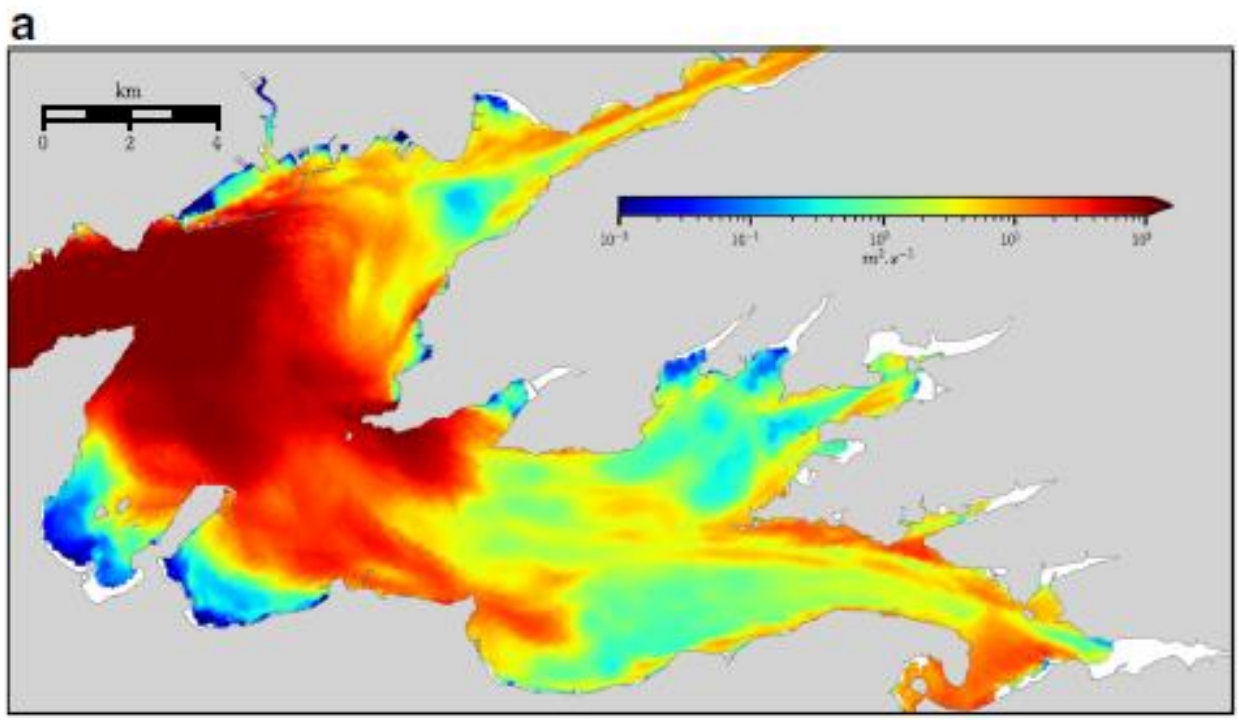

\section{b}

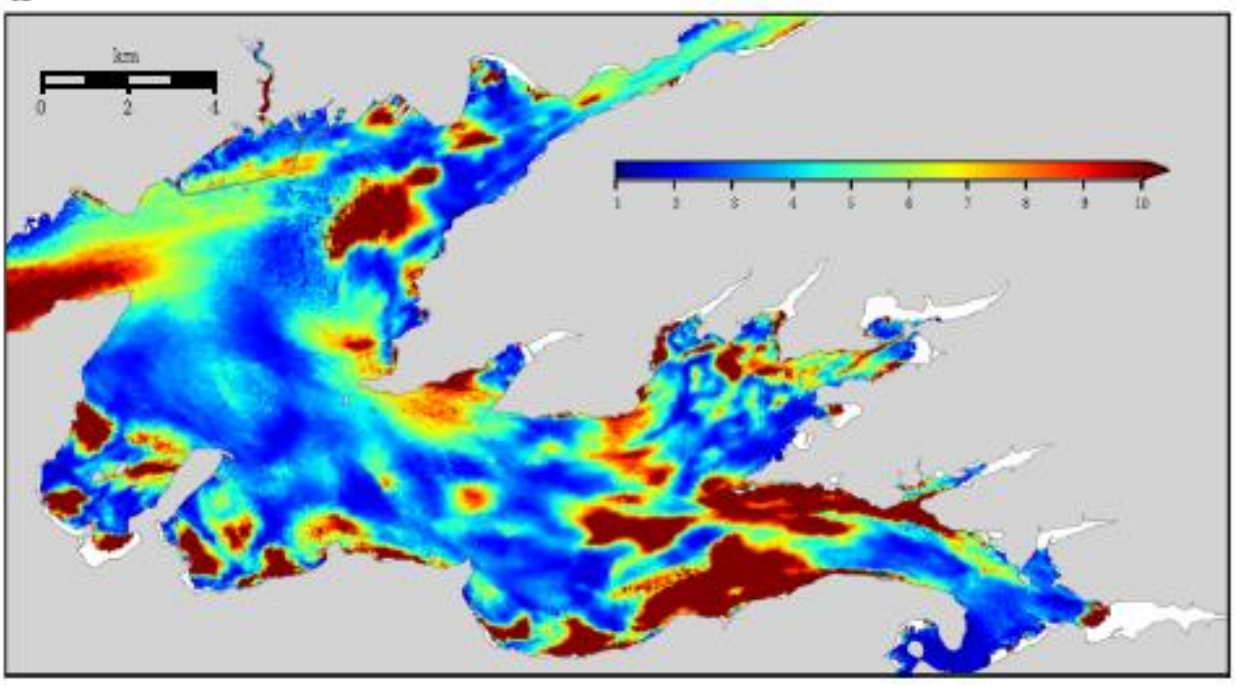


Fig. 5 Local diffusivity $K_{\xi}^{\prime}$ estimated in the same conditions as Fig. 4a but accounting for an additional dispersion (i.e. shear dispersion) horizontal dispersion of $K_{H}=1 \mathrm{~m}^{2} \mathrm{~s}^{-1}$. What is plotted is $K_{\xi}^{\prime}-K_{H}$

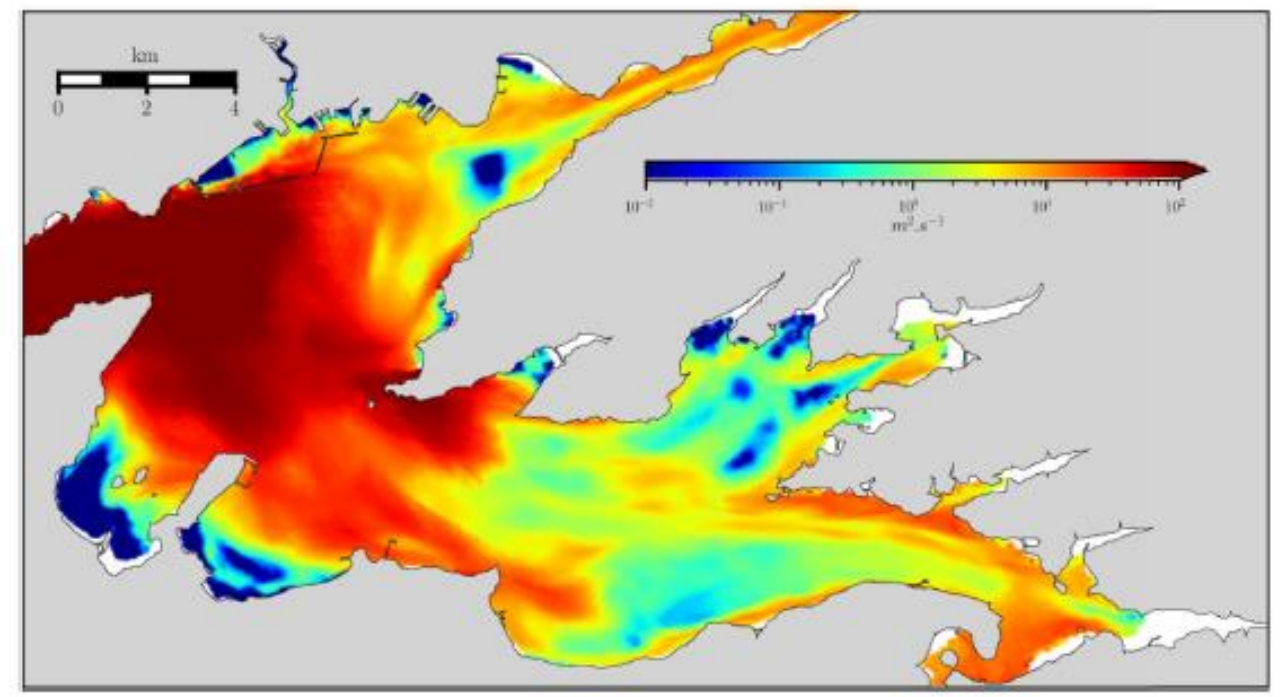

Fig. 6 (a) Tidal excursion length estimated for mean tide conditions without any meteorological forcing. (b) Median diffusivity $k_{z}$ from the MARS 3D model during a tidal cycle. (c) Probability density function expressed between the local diffusivity $K_{\xi}$ and the tidal excursion length. (d) Probability density function expressed between the local diffusivity $K_{\xi}$ and the diffusivity $k_{z}$.

a

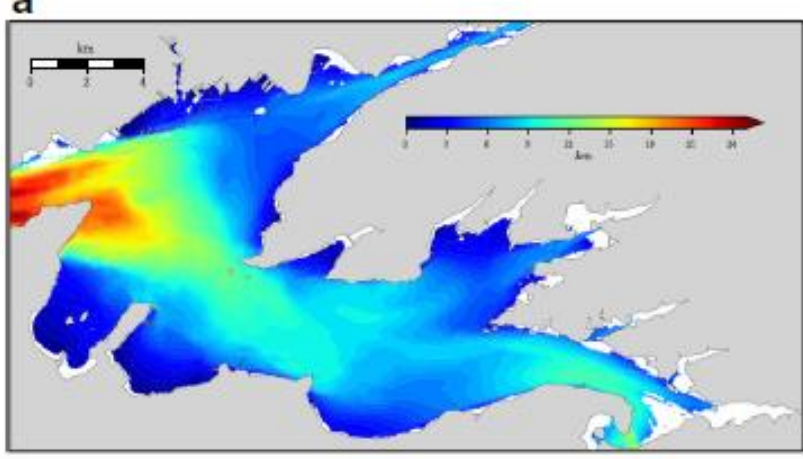

b

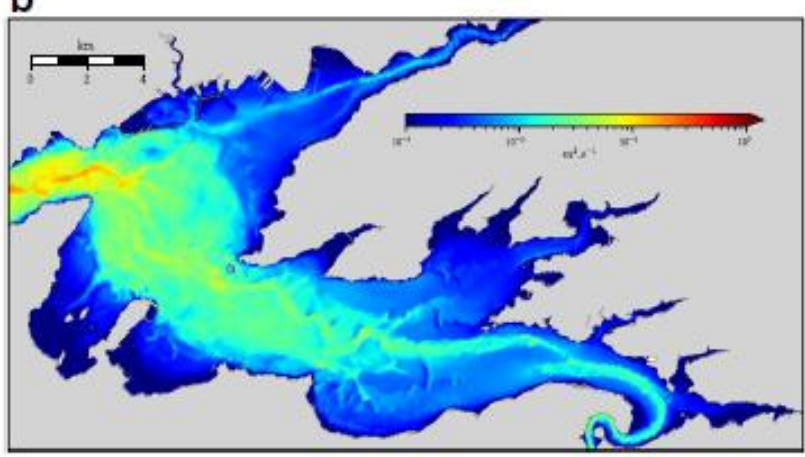

c

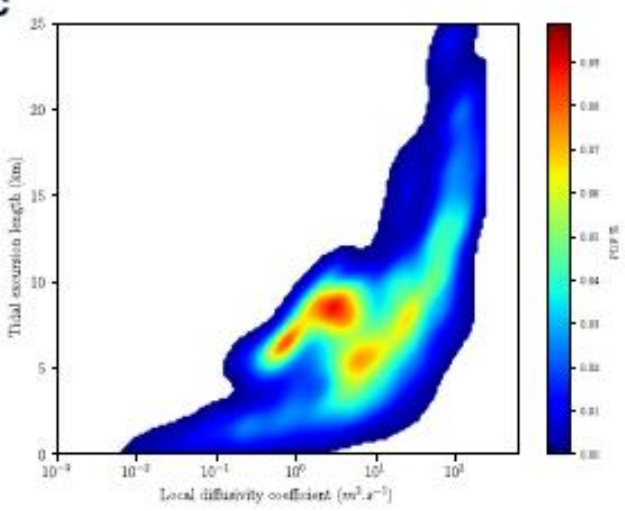

d

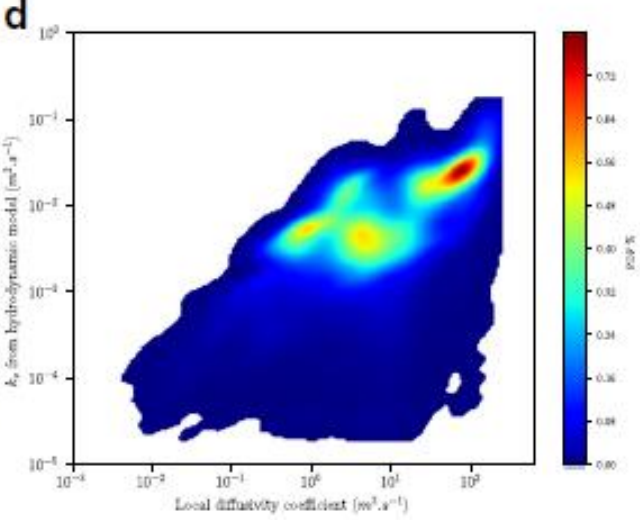


Fig. 7 Local diffusivity $\left(\mathrm{m}^{2} \cdot \mathrm{s}^{-1}\right)$ estimated for a perpetual mean tidal cycle after two (a), three (b) and four (c) tidal cycles.

\section{a}

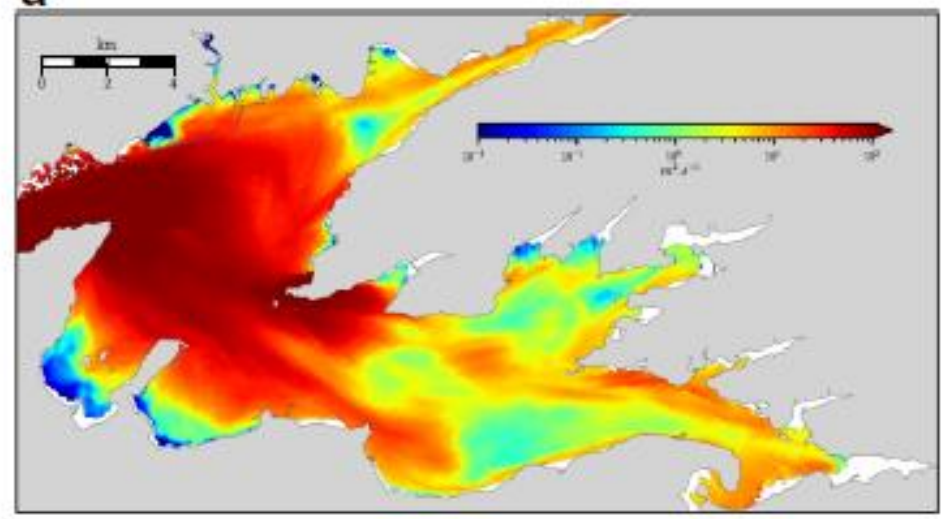

b

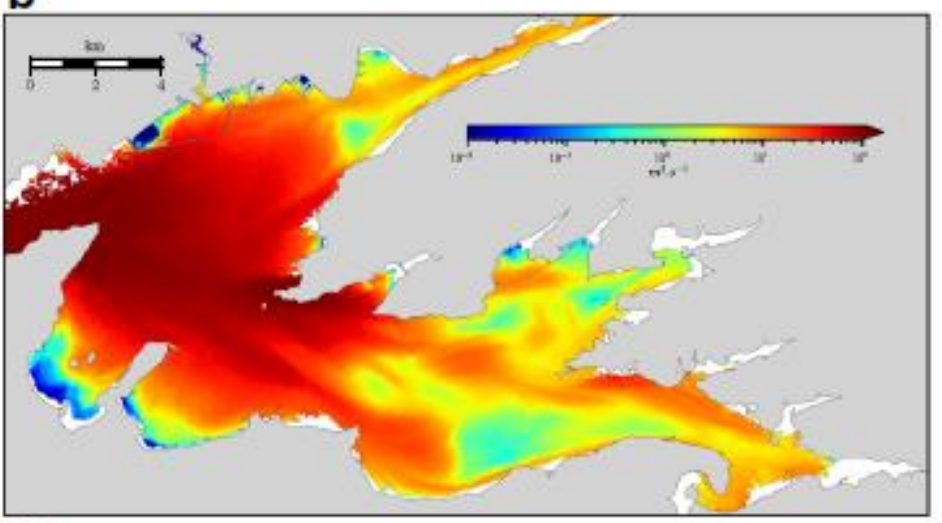

C

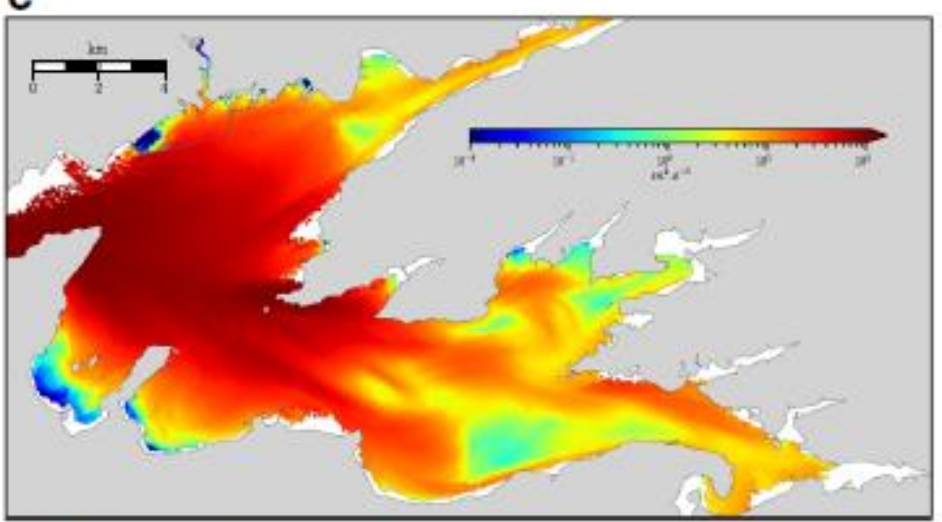

Fig. 7 Local diffusivity $\left(\mathrm{m}^{2} \mathrm{~s}^{-1}\right)$ estimated for a perpetual mean tidal cycle after two (a), three (b), and four (c) tidal cycles 
Fig. 8 Relative change in the local diffusivity with an increase of the radius (DES) compared with the $50 \mathrm{~m}$ DES. In each site, the coefficient is first scaled down by averaging its value over a $250 \mathrm{~m}$ square. Black crosses give the empirical relationship between the apparent diffusivity and the scale of diffusion $d^{1.15}$ from Okubo (1971)

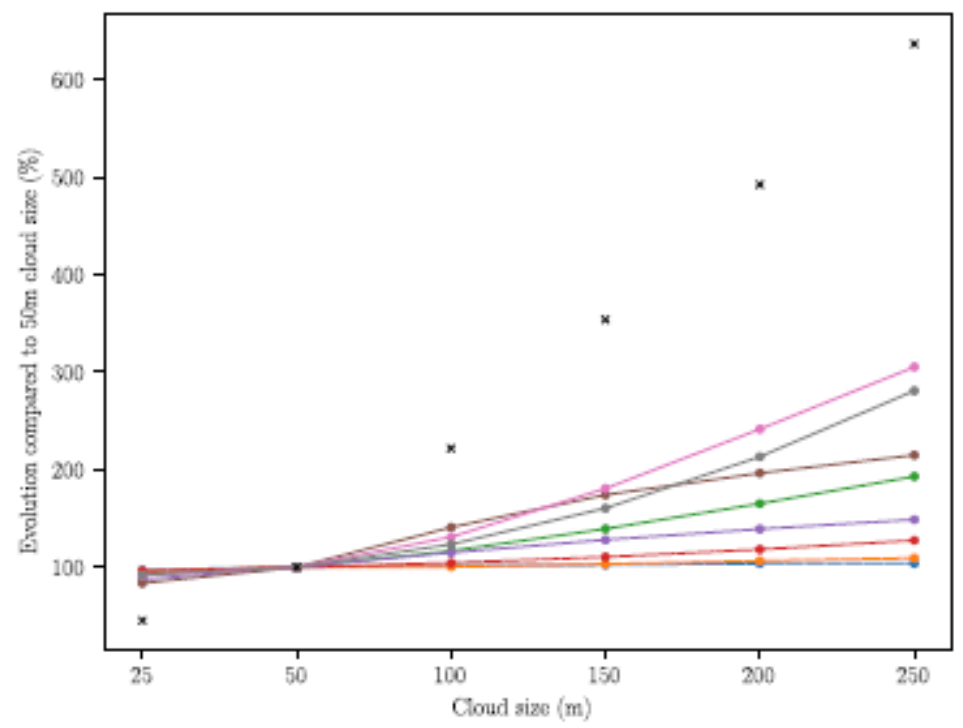

Fig. 9 (a) Local diffusivity estimation (Lagrangian repositioning) without any meteorological forcing over one neap tidal cycle (mean neap tidal range: $2.7 \mathrm{~m}$ ); (b) same as (a) but over a spring tidal cycle (mean spring tidal range: $5.9 \mathrm{~m}$ ).
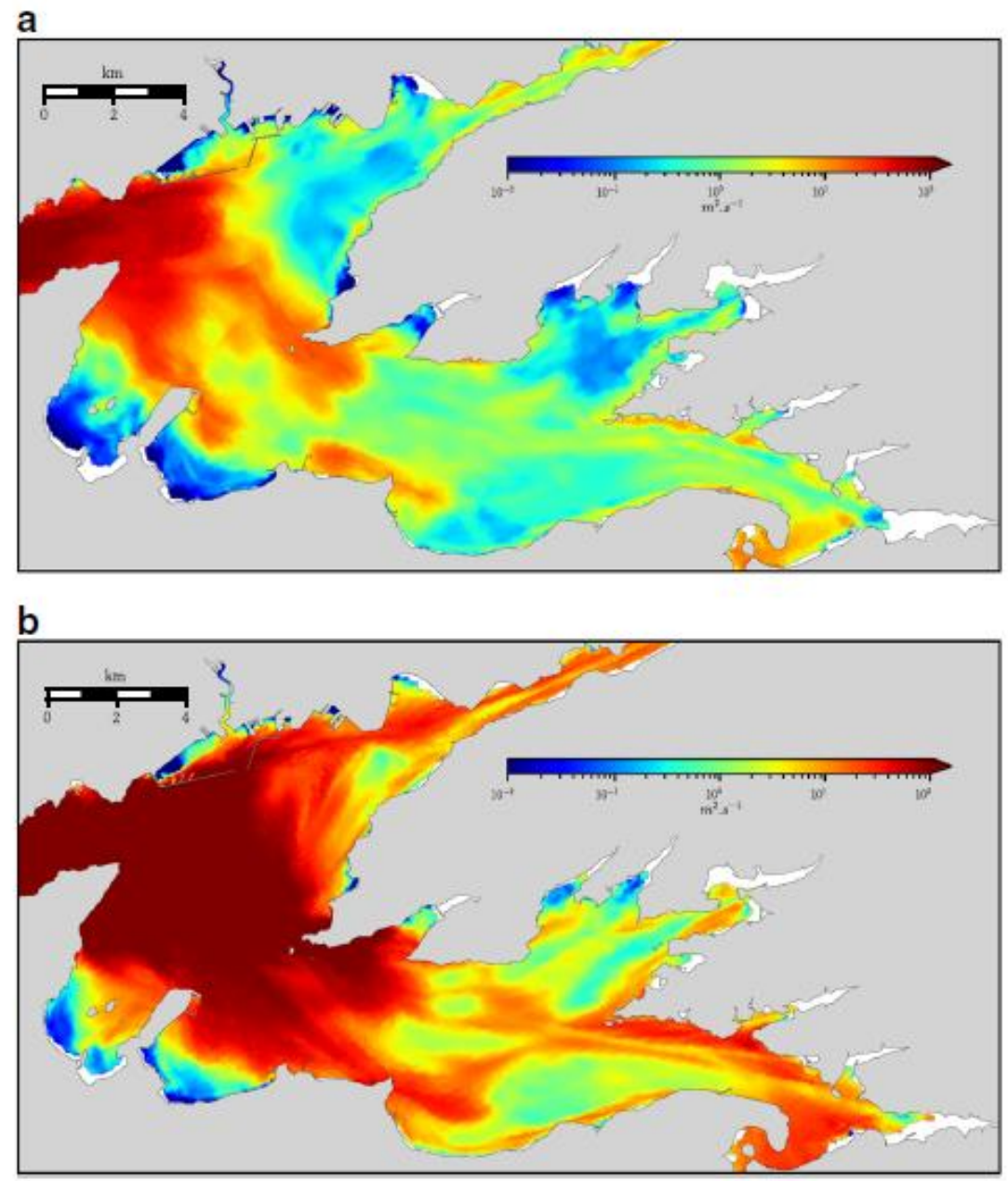
Fig. 10 Local diffusivity estimation with barycentric repositioning over one mean tidal cycle with a stationary wind of $5 \mathrm{~m} \cdot \mathrm{s}^{-1}$ (a) and $10 \mathrm{~m} \cdot \mathrm{s}^{-1}$ (c) from the north-east and the relative impact in \% (b) and (d) on the coefficient compared to the reference situation (Fig. 4a); the same for a stationary wind of $5 \mathrm{~m} . \mathrm{s}^{-1}$ (e) and $10 \mathrm{~m} . \mathrm{s}^{-1}$ (g) from the south-west and the relative impact in \% (f) and (h).
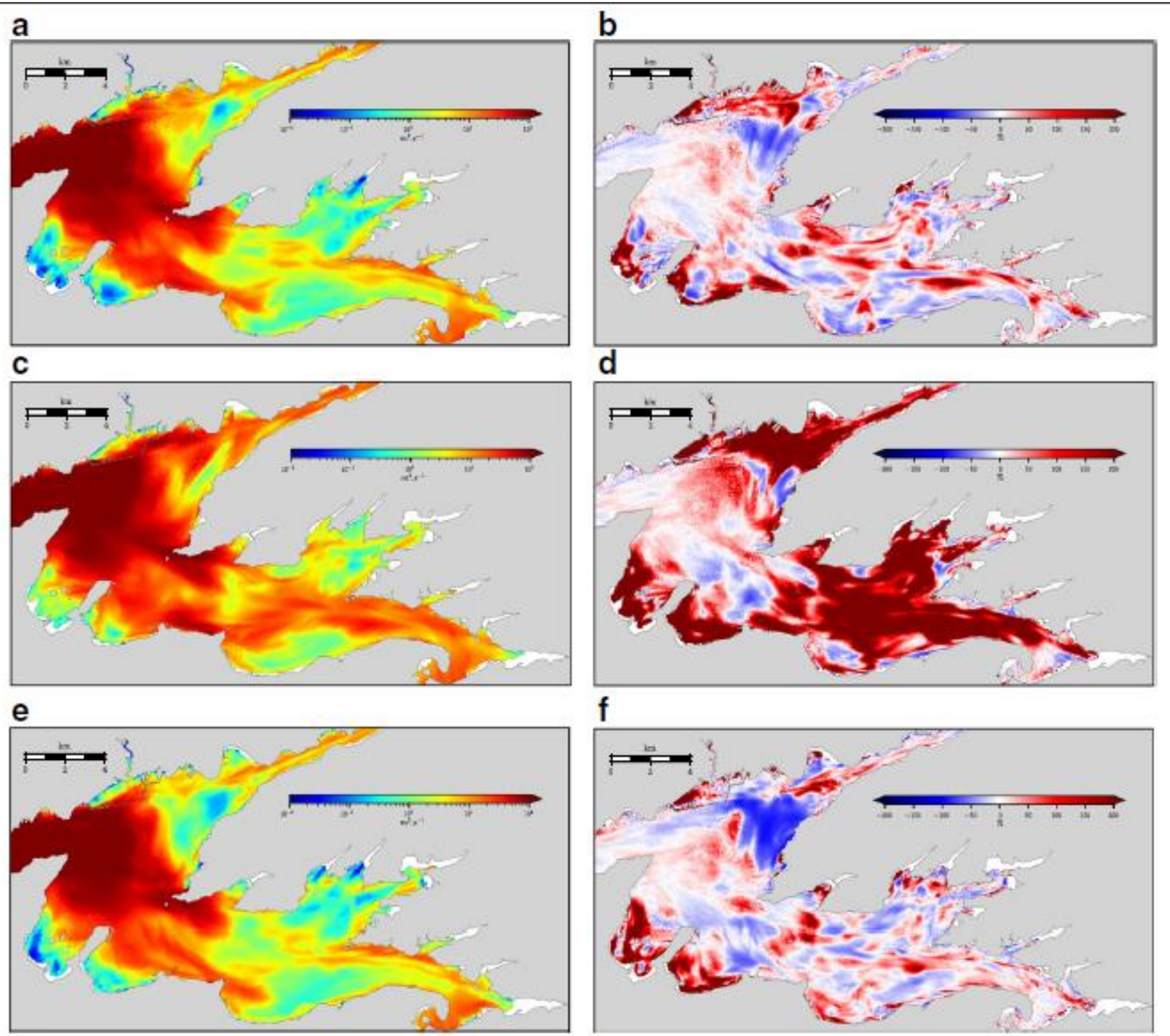

g
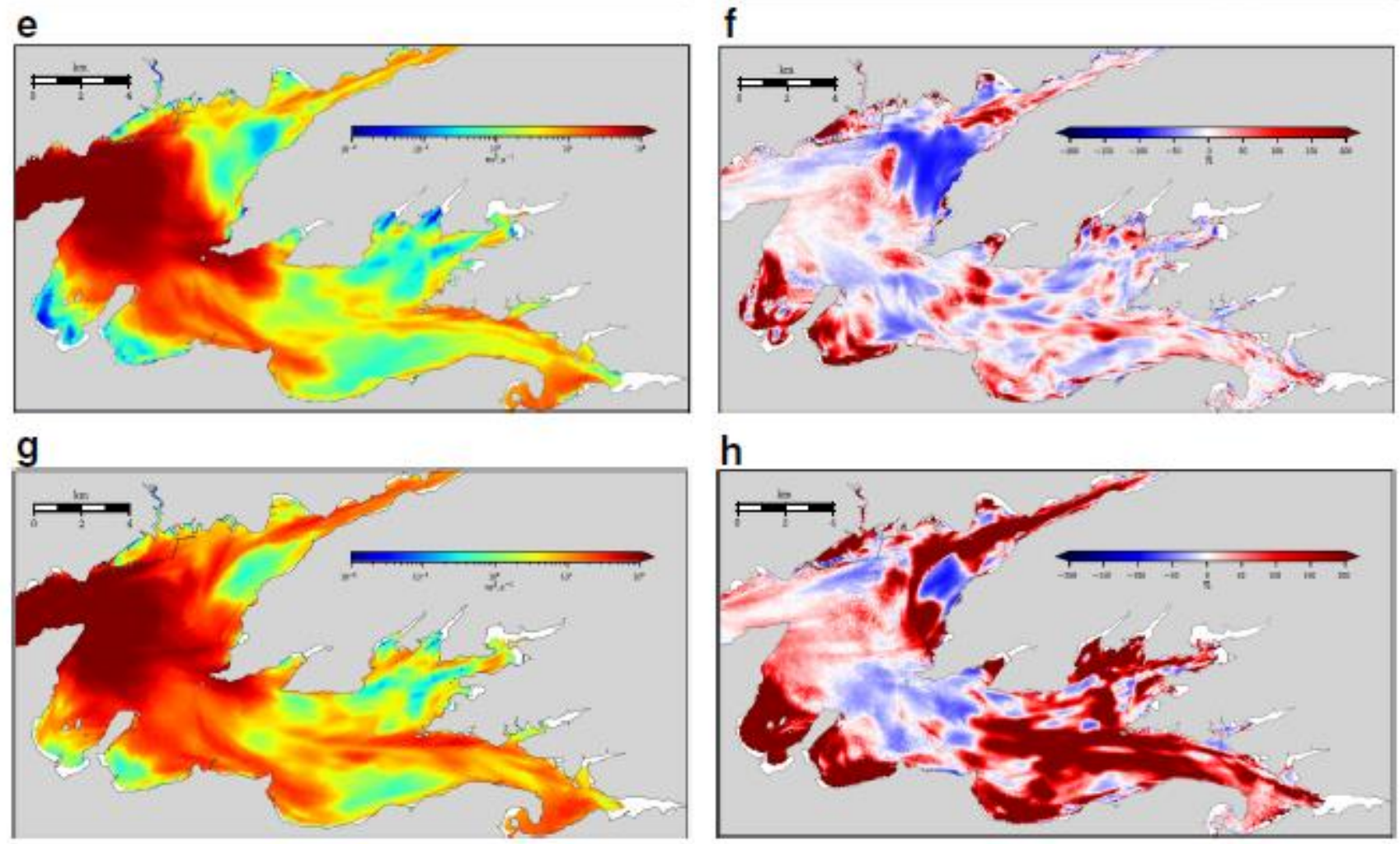